\title{
Zeros of conformal fields in any metric signature
}

\author{
Andrzej Derdzinski \\ Department of Mathematics, The Ohio State University, Columbus, OH 43210, USA \\ E-mail: andrzej@math.ohio-state.edu
}

\begin{abstract}
The connected components of the zero set of any conformal vector field, in a pseudo-Riemannian manifold of arbitrary signature, are shown to be totally umbilical conifold varieties, that is, smooth submanifolds except possibly for some quadric singularities. The singularities occur only when the metric is indefinite, including the Lorentzian case. This generalizes an analogous result in the Riemannian case, due to Belgun, Moroianu and Ornea (2010).
\end{abstract}

AMS classification scheme numbers: 53B30 


\section{Introduction}

A vector field $v$ on a pseudo-Riemannian manifold $(M, g)$ of dimension $n \geq 2$ is called conformal if, for some function $\phi: M \rightarrow \mathbb{R}$,

$$
£_{v} g=\phi g \text {, that is, in coordinates, } v_{j, k}+v_{k, j}=\phi g_{j k} .
$$

One then obviously has $\operatorname{div} v=n \phi / 2$. The class of conformal vector fields on $(M, g)$ includes Killing fields $v$, characterized by (11) with $\phi=0$.

Kobayashi [11] showed that, for any Killing vector field $v$ on a Riemannian manifold $(M, g)$, the connected components of the zero set of $v$ are mutually isolated totally geodesic submanifolds of even codimensions. Assuming compactness of $M$, Blair [5] established an analogue of Kobayashi's theorem for conformal vector fields, in which the word 'geodesic' is replaced by 'umbilical' and the codimension clause is relaxed for one-point connected components. Very recently, Belgun, Moroianu and Ornea [4] proved that Blair's conclusion remains valid in the noncompact case.

It is natural to ask what happens when the metric $g$ is indefinite. Questions about the structure of conformal fields arise in connection with some known open problems, such as those related to the pseudo-Riemannian Lichnerowicz conjecture [10].

The result of Belgun, Moroianu and Ornea [4], mentioned above, becomes false when repeated verbatim for indefinite metrics: even in pseudo-Euclidean spaces, connected components of the zero set of a conformal vector field may have quadric singularities (see Example 10.1 below). Such singularities, however, are the worst that can occur, aside from the fact that the codimension restriction has to be modified as well, cf. Example 10.1. More precisely, the following theorem is proved in Section 17. (A set in a vector space is called star-shaped if it is a union of line segments emanating from 0.)

Theorem A. Let $Z$ denote the zero set of a conformal vector field $v$ on a pseudoRiemannian manifold $(M, g)$ of dimension $n \geq 3$. Then every point $z \in Z$ has a neighborhood $U^{\prime}$ in $M$ such that, for some star-shaped neighborhood $U$ of 0 in $T_{z} M$, and some metric $g^{\prime}$ on $U^{\prime}$ conformal to $g$, the exponential mapping $\exp _{z}$ of $g^{\prime}$ at $z$ is defined on $U$ and maps $U$ diffeomorphically onto $U^{\prime}$, while $Z \cap U^{\prime}=\exp _{z}[E \cap U]$ for $E \subseteq T_{z} M$ which is

(a) a vector subspace of $T_{z} M$, or

(b) the set of all null vectors in a vector subspace $H \subseteq T_{z} M$.

The singular subset $\Delta$ of $Z \cap U^{\prime}$ equals $\exp _{z}\left[H \cap H^{\perp} \cap U\right]$ in case (ii), if the metric restricted to $H$ is not semidefinite, and $\Delta=\varnothing$ otherwise. The connected components of $\left(Z \cap U^{\prime}\right) \backslash \Delta$ are totally umbilical submanifolds of $(M, g)$, and their codimensions are even unless $\Delta=\varnothing$ and $Z \cap U^{\prime}$ is a null totally geodesic submanifold of $(M, g)$. In addition, $\operatorname{div} v$ is constant along each connected component of $Z$.

Remark 17.2 discusses the meaning of Theorem $\mathrm{A}$ in the Lorentzian case.

Theorem $\mathrm{A}$ does not extend to dimension 2. In the metric signature -+ the zero set of a conformal field $v$ may be quite pathological (Example 10.2), even though on a 
Riemannian surface $(M, g)$ such $v$ is locally holomorphic, and so its zero set is discrete or equal to $M$, cf. [4].

The argument in Sections 14 - 17, leading to Theorem A, concentrates - just as Belgun, Moroianu and Ornea did in [4] - on the case where a conformal vector field $v$ on a (pseudo-)Riemannian manifold $(M, g)$ has a zero at a point $z \in M$ satisfying one of the following two conditions, with $\phi$ as in (1) and $\nabla \phi_{z}$ denoting its gradient at $z$ :

$$
\begin{aligned}
& \text { a) } \phi(z) \neq 0, \\
& \text { b) } \phi(z)=0 \text { and } \nabla \phi_{z} \notin \nabla v_{z}\left(T_{z} M\right) .
\end{aligned}
$$

Here $\nabla v_{z}\left(T_{z} M\right)$ is the image of $\nabla v_{z}: T_{z} M \rightarrow T_{z} M$, the value at $z$ of the covariant derivative $\nabla v$ treated as the bundle morphism $\nabla v: T M \rightarrow T M$ which sends each vector field $w$ to $\nabla_{w} v$.

The use of (2, a) - (2,b) is crucial in view of the following result of Beig [3].

Theorem B. For a conformal vector field $v$ on a pseudo-Riemannian manifold $(M, g)$ with $\operatorname{dim} M \geq 3$ and a zero $z$ of $v$, the following two conditions are equivalent:

(i) z has a neighborhood $U^{\prime}$ such that $v$ restricted to $U^{\prime}$ is a Killing field on $\left(U^{\prime}, g^{\prime}\right)$, where $g^{\prime}$ is some metric on $U^{\prime}$ conformal to $g$,

(ii) $\phi(z)=0$ and $\nabla \phi_{z} \in \nabla v_{z}\left(T_{z} M\right)$, that is, neither (2. a) nor (2., b) holds at $z$.

Proof. See [6].

A point $z \in M$ is said to be essential [4] for a conformal vector field $v$ on $(M, g)$ if condition (i) in Theorem B fails to hold. Thus, by Theorem B, essential zeros of $v$ are precisely those zeros at which (2,a) or (2,b) is satisfied. On the other hand, points where $v \neq 0$ are never essential, cf. the lines preceding Lemma 9.1.

In proving Theorem $\mathrm{A}$ we are allowed, by Theorem $\mathrm{B}$, to make the additional assumption that (2, a) or (2,b) holds. In fact, if one has neither (2, a) nor (2, b), Theorem B reduces the problem to studying the zero set of a Killing field, which is always linearized by normal coordinates. Assertion (a) of Theorem A then follows, for $E=\operatorname{Ker} \nabla v_{z}$, with $g^{\prime}$ chosen as in Theorem B(i). (See Section 17])

On the other hand, if one of conditions (2,a) - (2, b) is satisfied, case (b) in Theorem $\mathrm{A}$ is a direct consequence of the following result, proved in Sections 15 - 16.

Theorem C. Let $Z$ be the zero set of a conformal vector field $v$ on a pseudo-Riemannian manifold $(M, g)$ of dimension $n \geq 3$. If $z \in Z$, while $\exp _{z}$ denotes the exponential mapping of $g$ at $z$, the function $\phi$ in (1) has one of the properties (2.a) - (2.,b), and $U$ is a sufficiently small star-shaped neighborhood of 0 in $T_{z} M$ mapped by $\exp _{z}$ diffeomorphically onto a neighborhood $U^{\prime}$ of $z$ in $M$, then $Z \cap U^{\prime}=\exp _{z}[C \cap H \cap U]$ for $H=\operatorname{Ker} \nabla v_{z} \cap \operatorname{Ker} d \phi_{z} \subseteq T_{z} M$ and the null cone $C=\left\{u \in T_{z} M: g_{z}(u, u)=0\right\}$.

The paper is organized as follows. Sections 2 - 11 contain preliminary material, including Theorem 7.5 derived from the Morse-Bott lemma (more on which below). The three lemmas in Section 12, which deal with the behavior of conformal fields along null geodesics, are then used in Section 13 to establish the relation $\exp _{z}[C \cap H \cap U] \subseteq Z \cap U^{\prime}$, 
one of the two opposite inclusions constituting the equality $Z \cap U^{\prime}=\exp _{z}[C \cap H \cap U]$ required in Theorem C. The proof of the remaining inclusion is split into Sections 15 and 16, corresponding to two separate cases, (2,a) and (2,b). In the former, limiting properties of geodesic segments joining points of $\exp _{z}[C \cap H \cap U]$ to other zeros of $v$ near $z$ are used to conclude that the other zeros cannot lie arbitrarily close to $z$. A similar argument provides a part of the proof in the latter case: phrased as Lemma 14.1. it shows that nearby zeros at which $\phi \neq 0$ would lead to connecting limits, in the sense of Section 5, for certain subsets of $Z$ near $z$, which are contained in $H$, but not in the nullspace of $H$. The final step is provided by Theorem 7.5, which states, first, that the existence of such connecting limits would contradict the algebraic structure of the second covariant derivative of $v$ at $z$, and, secondly, that nearby zeros with $\phi=0$ must all lie in $\exp _{z}[C \cap H \cap U]$.

\section{Manifolds and submanifolds}

Unless stated otherwise, manifolds and submanifolds are connected, submanifolds carry the subset topology, while tensor fields and mappings are, by definition, of class $C^{\infty}$. By vector-valued functions we mean mappings into vector spaces, with the latter always assumed to be finite-dimensional and real.

Given a vector-valued function $\beta$ on $I \times K$, where $K$ is a manifold and $I \subset \mathbb{R}$ an interval containing 0 , the Newton-Leibniz formula and integration by parts yield

$$
\begin{aligned}
\text { i) } \quad \beta(s, y) & =\beta(0, y)+s \int_{0}^{1} \beta_{s}(t s, y) d t \\
\text { ii) } \quad \beta(s, y) & =\beta(0, y)+\beta_{s}(0, y) s+s^{2} \int_{0}^{1}(1-t) \beta_{s s}(t s, y) d t
\end{aligned}
$$

for $s \in I$ and $y \in K$, with ()$_{s}=\partial / \partial s$. In particular, $\beta$ is smoothly divisible by the projection function $(s, y) \mapsto s$ if $\beta=0$ whenever $s=0$. Similarly, for a vector-valued function $\Psi: U \rightarrow \mathcal{T}$ on a neighborhood $U$ of a point $z$ in a vector space $W$, and any $x, y$ near $z$ in $W$, integrating $d[\Psi(x+t(y-x))] / d t$ from $t=0$ to $t=1$ we obtain

$$
\Psi(y)-\Psi(x)=D_{(x, y)}(y-x), \quad \text { with } D_{(x, x)}=d \Psi_{x}
$$

where the function $(x, y) \mapsto D_{(x, y)} \in \operatorname{Hom}(W, \mathcal{T})$ is given by $D_{(x, y)}=\int_{0}^{1} d \Psi_{x+t(y-x)} d t$.

Lemma 2.1. Let a vector-valued function $\beta$ on a manifold $N$ vanish at all points of a codimension-one submanifold $K$.

(a) If $z \in K$ and $d \beta_{z} \neq 0$, then $z$ has a neighborhood $U$ in $N$ such that $\beta \neq 0$ everywhere in $U \backslash K$.

(b) If $d \beta \neq 0$ everywhere in a set $\Xi \subseteq K$, then for some open subset $U$ of $N$ containing $\Xi$ we have $\beta \neq 0$ at all points of $U \backslash K$.

Proof. Let us replace $K$ with a smaller neighborhood of $z$ in $K$, if necessary, so as to identify a neighborhood of $z$ in $N$ with $I \times K$ and $K$ with $\{0\} \times K$, for $I$ as in (3). Since $d \beta_{z} \neq 0$, we have $\int_{0}^{1} \beta_{s}(0, y) d t=\beta_{s}(0, y) \neq 0$ in (3.i), for $y=z$. This yields (a), while (a) obviously implies (b). 
Given a submanifold $K$ of a manifold $M$, the normal bundle of $K$ is defined, as usual, to be the quotient vector bundle $\mathcal{N}=T_{K} M / T K$, where $T_{K} M$ is the restriction of $T M$ to $K$. A fixed torsionfree connection $\nabla$ on $M$ then gives rise to the second fundamental form of $K$, which is a section $b$ of $\operatorname{Hom}\left([T M]^{\odot 2}, \mathcal{N}\right)=\left[T^{*} M\right]^{\odot 2} \otimes \mathcal{N}$ (in other words, $b_{x}: T_{x} K \times T_{x} K \rightarrow \mathcal{N}_{x}$ is, at every $x \in K$, bilinear and symmetric). We have $b(\dot{x}, \dot{x})=\pi \nabla_{\dot{x}} \dot{x}$ whenever $t \mapsto x(t)$ is a curve in $K$, with $\pi: T M \rightarrow \mathcal{N}$ denoting the quotient projection.

Lemma 2.2. Let $b$ be the second fundamental form of a submanifold $K$ in a manifold $M$ endowed with a torsionfree connection $\nabla$.

(i) $b(\dot{x}, \dot{x})=0$ along any geodesic $t \mapsto x(t)$ of $\nabla$ which is contained in $K$.

(ii) If $z \in M$, a neighborhood $U$ of 0 in $T_{z} M$ is mapped by $\exp _{z}$ diffeomorphically onto a neighborhood of $z$ in $M$, and $K=\exp _{z}[V \cap U]$ for a vector subspace $V$ of $T_{z} M$, then $b_{z}=0$.

Proof. Assertion (i) is obvious from the formula $b(\dot{x}, \dot{x})=\pi \nabla_{\dot{x}} \dot{x}$, and (ii) from (i) for all the geodesics $x(t)=\exp _{z} t u$ with $u \in V$.

When $b=0$ identically, $K$ is said to be totally geodesic relative to $\nabla$. If $\nabla$ is the Levi-Civita connection of a pseudo-Riemannian metric $g$ on $M$ and $b=g_{K} \otimes u$ for some section $u$ of $\mathcal{N}$. where $g_{K}$ is the restriction of $g$ to $K$, one calls $K$ totally umbilical in $(M, g)$. This last property of $K$ is conformally invariant, since

$$
\text { changing } g \text { to } e^{-\tau} g \text { causes } b \text { to be replaced by } b+g_{K} \otimes \pi \nabla \tau / 2 \text {. }
$$

A null submanifold of a pseudo-Riemannian manifold $(M, g)$ is totally umbilical if and only if it is totally geodesic. The class of (unparametrized) null geodesics in $(M, g)$ is therefore a conformal invariant.

\section{Differentials and Hessians}

As before, vector-valued functions are mappings into finite-dimensional real vector spaces. For a fixed curve $t \mapsto x(t)$ in a manifold $M$ and a vector-valued function $f$ on $M$, we write

$$
\dot{f}=d[f(x(t))] / d t, \quad \ddot{f}=d^{2}[f(x(t))] / d t^{2} .
$$

Given a vector bundle $\mathcal{E}$ over a manifold $M$, a section $\psi$ of $\mathcal{E}$ defined on an open set $U \subseteq M$, and a point $z \in U$ at which $\psi_{z}=0$, the differential of $\psi$ at $z$ is the linear operator $\partial \psi_{z}: T_{z} M \rightarrow \mathcal{E}_{z}$ arising as the composition of the ordinary differential $d \psi_{z}: T_{z} M \rightarrow T_{z} \mathcal{E}$ and the projection $T_{z} \mathcal{E} \rightarrow \mathcal{E}_{z}$ coming from the natural identification $T_{z} \mathcal{E} \approx T_{z} M \oplus \mathcal{E}_{z}$. (Here $z \in M \subseteq \mathcal{E}$, with $M$ treated as the zero section embedded in the total space $\mathcal{E}$, and $\psi$ viewed as a mapping $M \rightarrow \mathcal{E}$.) The components of $\partial \psi_{z}$ relative to fixed local coordinates and a local trivialization of $\mathcal{E}$, defined around $z$, are $\partial_{j} \psi^{a}$, so that $\partial \psi_{z}=\nabla \psi_{z}$ for any connection $\nabla$ in $\mathcal{E}$. 
ExAmPLE 3.1. For $\mathcal{E}, M, \psi, U$ and $z$ as above, with $\psi_{z}=0$, let $n=\operatorname{dim} M$ and $r=\operatorname{rank} \partial \psi_{z}$. Then all zeros of $\psi$ near $z$ lie in some $(n-r)$-dimensional submanifold $N$ of $M$ having the property that $\operatorname{Ker} \partial \psi_{x} \subseteq T_{x} N$ whenever $x \in N$ and $\psi_{x}=0$.

To construct such $N$, we may start with an $r$-dimensional real vector space $W$ and a base-preserving bundle morphism $G$ from $\mathcal{E}$ into the product vector bundle $M \times W$. In other words, $G$ is a $W$-valued $C^{\infty}$ function on the total space $\mathcal{E}$ and its restriction $G_{x}$ to the fibre $\mathcal{E}_{x}$ is linear for each $x \in M$. We now choose $G$ so that $G_{z}$ sends the image $\partial \psi_{z}\left(T_{z} M\right)$ isomorphically onto $W$. The mapping $F: U \rightarrow W$ defined by $F(x)=G_{x} \psi_{x}$ has, at any $x \in U$ with $\psi_{x}=0$, the differential $d F_{x}=G_{x} \circ \partial \psi_{x}$. Applied to $x=z$, this shows that $F$ is a submersion at $z$ and, making $U$ smaller if necessary, we can simply set $N=U \cap F^{-1}(0)$.

ExAmPle 3.2. (a) In the case where $\mathcal{E}=T M$ and $z \in M$ is a zero of a vector field $v$ defined on a neighborhood of $z$, the differential $\partial v_{z}$ obviously coincides with the infinitesimal generator of the local flow of $v$ acting in $T_{z} M$.

(b) If $f: M \rightarrow W$ is a vector-valued function on a manifold $M$ and $d f_{z}=0$ at a point $z \in M$, the differential $\partial d f_{z}$ of $d f$ at $z$ is nothing else than the Hessian of $f$ at $z$. Here $d f$ is a section of the bundle $\mathcal{E}$ of $W$-valued 1 -forms on $M$. We will use the fact that, for a curve $t \mapsto x(t)$ in $M$ with $x(0)=z$,

$$
\ddot{f}(0)=\partial d f_{z}(u, u) \text { (notation of (마) ), where } u=\dot{x}(0) \text {. }
$$

REMARK 3.3. Let $N$ be the submanifold constructed in Example 3.1, with the corresponding $\mathcal{E}, M, \psi, U, z, n, r, G$ and $F$. Suppose that, in addition, $\mathcal{E}$ and $T M$ are endowed with connections, of which the latter is torsionfree, and both are denoted by $\nabla$, while $\xi$ is a fixed section of the dual bundle $\mathcal{E}^{*}$, and the function $Q: N \rightarrow \mathbb{R}$ is defined to be the restriction of $\xi(\psi)$ to $N$. Then

(i) $d Q_{x}=0$ at every $x \in N$ with $\psi_{x}=0$ and rank $\partial \psi_{x}=r$, including $x=z$,

(ii) in the case where $\xi_{z}$ vanishes on the image $\partial \psi_{z}\left(T_{z} M\right)$ (that is, $\left.\partial \psi_{z}\left(T_{z} M\right) \subseteq \operatorname{Ker} \xi_{z}\right)$, the Hessian of $Q$ at $z$ is given by $\partial d Q_{z}(u, u)=\xi(\theta(u, u))$ for all $u \in T_{z} N=$ Ker $\partial \psi_{z}$, with $\theta$ denoting the second covariant derivative of $\psi$ relative to the two connections and their tensor product: $\theta(u, w)=\left[\nabla_{u}(\nabla \psi)\right] w$ whenever $u, w \in T_{z} M$.

In fact, for a curve $t \mapsto x(t)$ in $N$ we have $\dot{Q}=\left(\xi_{a} \psi^{a}\right)^{\cdot}=\dot{x}^{j}\left(\xi_{a, j} \psi^{a}+\xi_{a} \psi^{a}{ }_{j}\right)$, (notation of (6) and the lines preceding Example 3.1, with commas standing for covariant derivatives). This gives (i), since for all $x$ in question the inclusion $\operatorname{Ker} \partial \psi_{x} \subseteq T_{x} N$ is an equality. If $x(0)=z$ and $\dot{x}(0)=u$, differentiating covariantly with respect to $t$ once again, at $t=0$, we obtain, from (7), $\partial d Q_{z}(u, u)=u^{j} u^{k} \xi_{a}(z) \psi^{a}{ }_{, j k}(z)$, as required; note that $\xi_{a} \psi^{a}{ }_{, j}=0$ at $t=0$ ( since $\left.\partial \psi_{z}\left(T_{z} M\right) \subseteq \operatorname{Ker} \xi_{z}\right)$, and $\psi^{a}{ }_{, j k}=\theta_{k j}^{a}$.

\section{Normal-coordinate neighborhoods and rigid geodesics}

As before, a subset of a vector space is said to be star-shaped if it is a union of line segments emanating from 0 . 
For a fixed connection $\nabla$ on a manifold $M$, a nontrivial $\nabla$-geodesic segment $\Gamma$ with endpoints $y, x$ will be called rigid if there exists an open subset $U^{\prime}$ of $M$ containing $\Gamma$ such that $\Gamma$ is the unique geodesic segment in $U^{\prime}$ joining $y$ to $x$. By a normal-coordinate neighborhood of a point $z \in M$ we mean any open set $U^{\prime} \subseteq M$ which is the $\exp _{z}$-diffeomorphic image of a star-shaped neighborhood $U$ of 0 in $T_{z} M$, with $\exp _{z}$ denoting the exponential mapping of $\nabla$ at $z$. Each point $x \in U^{\prime}$ then is joined to $z$ by a unique $\nabla$-geodesic segment $\Gamma$ contained in $U^{\prime}$ (and so $\Gamma$ is rigid).

Let $T M$ be the total space of the tangent bundle of $M$. Our convention is that, as a set, $T M=\left\{(x, w): x \in M\right.$ and $\left.w \in T_{x} M\right\}$. We identify $M$ and each tangent space $T_{x} M$, in the standard way, with subsets of $T M$ (the zero section and the fibre $\left.\{x\} \times T_{x} M\right)$, and say that a subset of $T M$ is radial if its intersection with each $T_{x} M$ is a (possibly empty) star-shaped set in $T_{x} M$. For a fixed connection $\nabla$ on $M$, the formula $\operatorname{Exp}(x, w)=\left(x, \exp _{z} w\right)$ defines a mapping from a radial open submanifold of $T M$, containing the zero section, into $M \times M$. In view of the inverse mapping theorem, Exp restricted to a suitable radial neighborhood $\Omega$ of any point $(z, 0)$ in the zero section is a diffeomorphism onto a neighborhood $\Omega^{\prime}$ of $(z, z)$ in $M \times M$. We will call a normalcoordinate neighborhood $U^{\prime}$ of z subconvex if $U^{\prime} \times U^{\prime} \subseteq \Omega^{\prime}$ for some such $\Omega$ and $\Omega^{\prime}$. More precisely, we will treat $\Omega$ as a "part of the structure" of the subconvex normalcoordinate neighborhood $U^{\prime}$ of $z$, so that, whenever $(x, y)=\operatorname{Exp}(x, w) \in U^{\prime} \times U^{\prime}$ with $(x, w) \in \Omega$, we may refer to the curve $[0,1] \ni t \mapsto \operatorname{Exp}(x, t w)$ as the rigid geodesic segment in $M$ joining $x$ to $y$.

REMARK 4.1. If $\nabla$ is the Levi-Civita connection of a pseudo-Riemannian metric $g$ on $M$, the class of null geodesic segments in $(M, g)$ depends only on the underlying conformal structure of $g$. (See the end of Section 2.) On the other hand, for any conformal vector field $v$ on $(M, g)$, the local flow of $v$ consists of conformal diffeomorphisms. Consequently, if $v=0$ at both endpoints of a rigid nontrivial null geodesic segment $\Gamma$ in $(M, g)$, then, due to uniqueness of $\Gamma$, the local flow of $v$ maps $\Gamma$ into itself.

\section{Connecting limits and radial limit directions}

Suppose that $M$ is a manifold, $z \in M$, and $L$ is a line through 0 in $T_{z} M$. Let us also fix a norm || in $T_{z} M$ and a neighborhood $U$ of 0 in $T_{z} M$ along with a diffeomorphism $\Psi: U \rightarrow U^{\prime}$ onto a neighborhood $U^{\prime}$ of $z$ in $M$ such that $\Psi(0)=z$ and $d \Psi_{0}=\mathrm{Id}: T_{z} N \rightarrow T_{z} N$. We call $L$ a connecting limit for a pair of sequences $x_{j}, y_{j} \in M$, $j=1,2, \ldots$, both converging to $z$ and having $x_{j} \neq y_{j}$ whenever $j$ is sufficiently large, if, for all but finitely many $j$, and $u_{j}, w_{j} \in U$ such that $\Psi\left(u_{j}\right)=x_{j}, \Psi\left(w_{j}\right)=y_{j}$, the limit of the sequence $\left(w_{j}-u_{j}\right) /\left|w_{j}-u_{j}\right|$ exists and spans $L$.

For such $M, z$ and $x_{j}, y_{j}$, neither $L$ itself nor the fact of its existence depends on the choice of || and $\Psi$. This is obvious for || , and for $\Psi$ it amounts to the following claim: if, in addition, $M$ is a neighborhood of $z=0$ in a vector space $W$, so that $T_{z} M=W$, and $\left(y_{j}-x_{j}\right) /\left|y_{j}-x_{j}\right| \rightarrow u \in W$ as $j \rightarrow \infty$, then, for any diffeomorphism $\Psi$ with the stated properties, $\left[\Psi\left(y_{j}\right)-\Psi\left(x_{j}\right)\right] /\left|\Psi\left(y_{j}\right)-\Psi\left(x_{j}\right)\right| \rightarrow u$. 
To verify the italicized statement, note that, for $x, y$ near 0 in $W$, if one writes $x=x_{j}, y=y_{j}$, the assumption that $(y-x) /|y-x| \rightarrow u$ gives, by (4), $[\Psi(y)-\Psi(x)] /|y-x|=D_{(x, y)}[(y-x) /|y-x|] \rightarrow D_{(0,0)} u=u$. Since $[\Psi(y)-\Psi(x)] /|y-x|$ tends to the || -unit vector $u$, so does the sequence $[\Psi(y)-\Psi(x)] /|\Psi(y)-\Psi(x)|$ obtained by normalizing $[\Psi(y)-\Psi(x)] /|y-x|$, as required.

REMARK 5.1. Given $M, z, L$ as above, let $L \subset T_{z} M$ be the connecting limit for a pair of sequences $x_{j}, y_{j}$ with $x_{j} \neq y_{j}$, converging to $z$. Then

(i) $L \subseteq T_{z} N$ if $N$ is a submanifold of $M$ and $x_{j}, y_{j} \in N$ for all $j$,

(ii) $L \subseteq \operatorname{Ker} \partial \psi_{z}$ whenever $\psi\left(x_{j}\right)=\psi\left(y_{j}\right)=0$ for all $j$ and some section $\psi$ of a vector bundle over $M$.

In fact, we obtain (ii) by identifying a neighborhood $U^{\prime}$ of $z$ in $M$ with a neighborhood of $z=0$ in the vector space $W=T_{z} M$ and trivializing the bundle over $U^{\prime}$, so that $\psi$ becomes a vector-valued function $\left(\psi^{1}, \ldots, \psi^{q}\right): U^{\prime} \rightarrow \mathbb{R}^{q}$. Vanishing of each $\psi^{a}, a=1, \ldots, q$, at both points $x_{j}, y_{j}$ implies that $\dot{x}_{j}\left(t_{j}^{a}\right) \in \operatorname{Ker} d \psi_{y}^{a}$, where $x_{j}(t)=x_{j}+t\left(y_{j}-x_{j}\right)$ and $y=x_{j}\left(t_{j}^{a}\right)$ for each fixed $a$ and some sequence $t_{j}^{a} \in(0,1)$, $j=1,2, \ldots$. The convergence relation $\mathbb{R}\left(y_{j}-x_{j}\right) \rightarrow L$ now yields $L \subseteq$ Ker $d \psi_{z}^{a}$ for each $a$, and (ii) follows. Now (ii) yields (i), since $N=\psi^{-1}(0)$ for a vector-valued function $\psi$ which is a submersion onto a neighborhood of 0 in a vector space.

In the following lemma, which will be needed in Section 14, convergence of tangent directions refers to the appropriate Grasmannian bundle, and can also be interpreted as convergence in $T M$ of suitably normalized spanning vectors. For the definitions of subconvexity and rigidity, see Section 4

Lemma 5.2. Suppose that $U^{\prime}$ is a subconvex normal-coordinate neighborhood of a point $z$ in a manifold $M$ with a connection $\nabla$, a line $L$ through 0 in $T_{z} M$ is the connecting limit for a pair of sequences $x_{j}, y_{j} \in U^{\prime}, j=1,2, \ldots$, both converging to $z$, with $x_{j} \neq y_{j}$ for all $j$, and $[0,1] \ni t \mapsto x_{j}(t)$ denotes the rigid $\nabla$-geodesic segment joining $x_{j}$ to $y_{j}$ in $U^{\prime}$. Then $x_{j}(t) \rightarrow z$ and $\mathbb{R} \dot{x}_{j}(t) \rightarrow L$ as $j \rightarrow \infty$, uniformly in $t \in[0,1]$.

Proof. For $\Omega$ associated with $U^{\prime}$ as in Section 4 , $(x, w) \in \Omega$, and any $t \in[0,1]$, let us set $x(t)=\exp _{x} t w$. Then $(x, x(t))=\operatorname{Exp}(x, t w)$, cf. Section 4, and so the preimage of $(0, \dot{x}(t)) \in T_{(x, x(t))}(M \times M)$ under the differential of Exp at $(x, t w) \in T M$, is, obviously, the vector $(x, w) \in\{x\} \times T_{x} M=T_{(x, t w)}\left(\{x\} \times T_{x} M\right) \subseteq T_{(x, t w)}(T M)$, independent (under this identification) of $t$.

Passing to a subsequence, if necessary, we may assume that $\mathbb{R} \dot{x}_{j}(0) \rightarrow L^{\prime}$ as $j \rightarrow \infty$ for some line $L^{\prime} \subseteq T_{z} N$. Since $\left(x_{j}(0), x_{j}(t)\right)=\operatorname{Exp}\left(x_{j}(0), t w_{j}\right)$ for $w_{j}=\dot{x}_{j}(0)$, the relations $x_{j}(t) \rightarrow z$, where $t=0,1$, and $\mathbb{R} \dot{x}_{j}(0) \rightarrow L^{\prime}$ amount to $\left(x_{j}(0), w_{j}\right) \rightarrow(z, 0)$ and $\left(x_{j}(0), c_{j} w_{j}\right) \rightarrow(z, u)$ in $T M$ with suitable $c_{j} \in(0, \infty)$ and a vector $u \in L^{\prime} \backslash\{0\}$. The former relation clearly gives $\left(x_{j}(0), t w_{j}\right) \rightarrow(z, 0) \in T M$, and the latter, combined with the remark about independence of $t$ made in the last paragraph, implies that $\mathbb{R} \dot{x}_{j}(t) \rightarrow L^{\prime}$. In both cases, the convergence is uniform in $t$. 
Let us now identify $U^{\prime}$ with a neighborhood of 0 in a vector space $W$, which also trivializes $T M$ over $U^{\prime}$, and fix a norm $\|$ in $W$. Omitting the subscript $j$ in $x_{j}, y_{j}$ and $w_{j}=\dot{x}_{j}(0)$, we thus have $y=\exp _{x} w$ and $w /|w| \rightarrow u$ for some vector $u$ spanning $L^{\prime}$. From (44) for $\Psi=\exp _{x}$ we now obtain $(y-x) /|w|=[\Psi(w)-\Psi(0)] /|w| \rightarrow u$ (cf. the lines preceding Remark 5.1), and, again, convergence of $(y-x) /|w|$ to the || -unit vector $u$ implies the same for the normalized sequence $(y-x) /|y-x|$. Thus, $L^{\prime}$ is the connecting limit for the pair $x_{j}, y_{j}$, so that $L^{\prime}=L$. Since this happens for the limit $L^{\prime}$ of any convergent subsequence of $\mathbb{R} \dot{x}_{j}(0)$, our assertion follows.

Let $z$ be a point in a manifold $M$. If $X, Y \subseteq M$, we define $\mathbb{L}_{z}(X, Y)$ to be the set of all connecting limits for pairs $x_{j}, y_{j}$ of sequences in $M$ such that $x_{j}, y_{j}$ both converge to $z$, while $x_{j} \in X, y_{j} \in Y$ and $x_{j} \neq y_{j}$ for all $j$. By radial limit directions of a subset $Z \subseteq M$ at a point $z \in M$ we mean elements of $\mathbb{L}_{z}(\{z\}, Z)$. Radial limit directions of a submanifold $N \subseteq M$ at a point $z \in N$ are the same as lines through 0 in $T_{z} N$, as one sees choosing the diffeomorphism $\Psi$ used to define connecting limits in such a way that it maps a neighborhood of 0 in $T_{z} N \subseteq T_{z} M$ into $N$.

\section{Quadratic forms}

In this section all vector spaces are finite-dimensional and real. Given a symmetric bilinear form $\langle$,$\rangle in a vector space W$, we will denote by $C=\{x \in W:\langle x, x\rangle=0\}$ its null cone, and by $V^{\perp}=\{x \in W:\langle x, \cdot\rangle=0$ on $V\}$ the $\langle$,$\rangle -orthogonal complement of$ a vector subspace $V \subseteq W$. Thus, $W^{\perp}$ is the nullspace of $\langle$,$\rangle . The quadratic function$ $Q: W \rightarrow \mathbb{R}$ corresponding to $\langle$,$\rangle is given by Q(x)=\langle x, x\rangle$, and so its differential at any $x \in W$ is $d Q_{x}=2\langle x, \cdot\rangle$. Consequently,

the nullspace $W^{\perp}$ coincides with the set of critical points of $Q$.

REMARK 6.1. Let a symmetric bilinear form $\langle$,$\rangle in a vector space W$ be semidefinite. Then $\langle$,$\rangle satisfies the Schwarz inequality \langle x, y\rangle^{2} \leq\langle x, x\rangle\langle y, y\rangle$ for $x, y \in W$. (In fact, changing the sign of $\langle$,$\rangle if necessary so as to make it positive semidefinite, we$ can approximate it with positive-definite forms $\langle\rangle+,\varepsilon\langle,\rangle_{+}$, where $\varepsilon>0$ and $\langle,\rangle_{+}$ is positive definite.) Consequently, its null cone $C$ coincides with its nullspace $W^{\perp}$, and so $C$ is a vector subspace of $W$. Thus, $C$ is a (singularity-free) submanifold of codimension $\operatorname{rank}\langle$,$\rangle in W$.

REMARK 6.2. Let $C$ be the null cone and $W^{\perp}$ the nullspace of a symmetric bilinear form $\langle$,$\rangle in a vector space W$ which is not semidefinite. Then

(a) the set of singular points of $C$ is nonempty, and coincides with $W^{\perp}$,

(b) the nonsingular subset $C \backslash W^{\perp}$ is dense in $C$,

(c) the connected components of $C \backslash W^{\perp}$ are codimension-one submanifolds of $W$,

(d) for $y \in C$, denoting by $E_{y} \subseteq W$ the union of all radial limit directions of $C$ at $y$ (defined at the end of Section (5), we have $E_{y}=C$ if $y \in W^{\perp}$, and $E_{y}=T_{y} C=y^{\perp}$ 
if $y \in C \backslash W^{\perp}$, so that in the former case $E_{y}$ spans $W$, and in the latter $E_{y}$ is a codimension-one subspace of $W$.

Namely, we have (c) since 0 is a regular value of the function $u \mapsto\langle u, u\rangle$ restricted to $W \backslash W^{\perp}$ (the differential of which, at any $u \in W$, is $2\langle u, \cdot\rangle$ ). Also, $\langle$,$\rangle descends to$ a symmetric bilinear form in $W / W^{\perp}$ which is nondegenerate and indefinite, so that it has nonzero null vectors lying arbitrarily close to 0 , and (b) follows.

Next, $C$ spans $W$. In fact, we may choose a $\langle$,$\rangle -orthogonal basis w_{j}, u_{a}, v_{\mu}$, where the index $j$ (or $a$, or $\mu$ ) ranges between 1 and some $i_{+} \geq 1$ (or, some $i_{-} \geq 1$, or, respectively, some $k \geq 0$ ), while $\left\langle w_{j}, w_{j}\right\rangle=1=-\left\langle u_{a}, u_{a}\right\rangle$ and $\left\langle v_{\mu}, v_{\mu}\right\rangle=0$. Thus, $W$ has a basis of $\langle$,$\rangle -null vectors, formed by all w_{1}-u_{a}$, all $u_{1}+w_{j}$, and all $v_{\mu}$. Now (d) is immediate from (c) and the final sentence of Section 5, while (a) is an obvious consequence of $(\mathrm{d})$.

REMARK 6.3. If $($,$) is a nondegenerate symmetric bilinear form on a vector space W$ and $\Sigma=\{u \in W:|u|=1\}$ denotes the unit sphere of a fixed Euclidean norm || in $W$, then 0 is a regular value of the function $\Sigma \ni u \mapsto(u, u)$.

More precisely, the differential of this function at any $u \in \Sigma$ is $2(u, \cdot)$ restricted to $T_{u} \Sigma$, which is nonzero when $(u, u)=0$, or else $u$ would be $($,$) -orthogonal to u$ as well as to $T_{u} \Sigma$, and hence to the whole space $W=\mathbb{R} u \oplus T_{u} \Sigma$.

\section{Some consequences of the Morse-Bott lemma}

The following result is often referred to as the Morse-Bott lemma.

Lemma 7.1. Suppose that a submanifold $K$ of a manifold $N$ consists of critical points of a function $Q: N \rightarrow \mathbb{R}$, while $z \in K$ and $Q(z)=0$. If for the Hessian $\partial d Q_{z}$ we have $\operatorname{rank} \partial d Q_{z} \geq \operatorname{dim} N-\operatorname{dim} K$, then there exists a diffeomorphism $\Psi$ of a neighborhood $U$ of 0 in $T_{z} N$ onto a neighborhood $U^{\prime}$ of $z$ in $M$ such that $\Psi(0)=z$ and $d \Psi_{0}=\mathrm{Id}: T_{z} N \rightarrow T_{z} N$, while $Q \circ \Psi$ equals the restriction to $U$ of the quadratic function of $\partial d Q_{z}$, and $K \cap U^{\prime}=\Psi(V \cap U)$, where $V \subseteq T_{z} M$ is the nullspace of $\partial d Q_{z}$.

Proof. See [2]. Note that, as the nullspace of $\partial d Q_{x}$ contains $T_{x} K$ whenever $x \in K$, the inequality assumed about $\operatorname{rank} \partial d Q_{x}$ at $x=z$ is actually an equality, not just at $z$, but also at all nearby $x \in K$. Also, the requirement that $d \Psi_{0}=\mathrm{Id}$, not explicitly mentioned in [2], can easily be realized, as it satisfied when $f$ is already diffeomorphically identified with a quadratic function.

Consider now a subset $Z$ of a manifold $N$, a point $z \in Z$ and a symmetric bilinear form (, ) in $T_{z} N$. We will call $Z$ a quadric at $z$ in $N$ modelled on $($, ) if there exists a diffeomorphism $\Psi$ of a neighborhood $U$ of 0 in $T_{z} N$ onto a neighborhood $U^{\prime}$ of $z$ in $M$ such that $\Psi(0)=z$ and $d \Psi_{0}=\operatorname{Id}: T_{z} N \rightarrow T_{z} N$, a s well as $Z \cap U^{\prime}=\Psi(C \cap U)$, where $C=\left\{u \in T_{z} N:(u, u)=0\right\}$ is the null cone of $($,$) .$

We may now rephrase one immediate consequence of Lemma 7.1 as follows. 
Lemma 7.2. Under the hypotheses of Lemma 17.1, the zero set $Z=Q^{-1}(0)$ is a quadric at $z$ in $N$ modelled on $\partial d Q_{z}$.

REMARK 7.3. A finite-dimensional real vector space with a fixed symmetric bilinear form $($, ) can always be decomposed into a (, )-orthogonal direct sum $W \oplus V$ of subspaces such that $($,$) is nondegenerate on W$ and $()=$,0 on $V$. Consequently, $V$ is the nullspace of $($,$) . Denoting by \Sigma$ the || -unit sphere around 0 in $W$, for a fixed Euclidean norm || in $W \oplus V$, and by $Q$ the quadratic function of $($,$) , we clearly have$ $Q(s u+x)=s^{2}(u, u)$ whenever $(s, u, x) \in \mathbb{R} \times \Sigma \times V$. Every neighborhood of 0 in $W \oplus V$ contains a smaller neighborhood of the form $B \oplus K=\{y+x: y \in B, x \in K\}$, where $B \subseteq W$ is the open ||-ball in $W$ of some radius $\varepsilon>0$, centered at 0 , and $K$ is a neighborhood of 0 in $V$.

The next lemma will be used in the proof of Theorem 7.5 .

Lemma 7.4. If $Y, Y^{\prime}$ are quadrics at a point $z$ in a manifold $P$, both modelled on the same symmetric bilinear form (, ) in $T_{z} P$, and $Y \subseteq Y^{\prime}$, then $U^{\prime} \cap Y=U^{\prime} \cap Y^{\prime}$ for some neighborhood $U^{\prime}$ of $z$ in $P$.

Proof. In the case where $($,$) is semidefinite, Y$ and $Y^{\prime}$ are submanifolds of codimension $\operatorname{rank}($,$) in P$ (see Remark 6.1), and our claim follows from the inverse mapping theorem applied to the inclusion $Y \rightarrow Y^{\prime}$.

Suppose now that $($,$) is not semidefinite. Using the notations and identifications$ introduced in Remark 7.3, we think of $P$ as a neighborhood of 0 in $T_{z} P=W \oplus V$ having the form $B \oplus K$, with $Y$ and $Y^{\prime}$ equal to the zero sets of the quadratic function $Q$ of (, ) and, respectively, of the function $Q^{\prime}$ obtained as the composite of $Q$ with a diffeomorphism between $B \oplus K$ and a neighborhood of 0 in $W \oplus V$, whose value and differential at 0 are 0 and Id. The Hessians of $Q$ and of $Q^{\prime}$ thus both equal 2(, ), while, by (8), the neighborhood $K$ of 0 in $V$ appearing in the equality $P=B \oplus K$ is precisely the set of critical points of $Q$, that is, singular points of $Y$ (see Remark 6.2(a)). In view of the characterization of singular and nonsingular points of a quadric, given in the final clause of Remark 6.2(d), all singular points of $Y$ are also singular in $Y^{\prime}$, so that $K$ must consist of critical points, as well as zeros, of $Q^{\prime}$.

For the open set $\Omega=(-\varepsilon, \varepsilon) \times \Sigma \times K$ in $\mathbb{R} \times \Sigma \times V$, where $\varepsilon$ is the radius of $B$ (cf. Remark 7.3) and the function $\beta: \Omega \rightarrow \mathbb{R}$ given by $\beta(s, u, x)=Q^{\prime}(s u+x$ ), we thus have $\beta(0, u, x)=\beta_{s}(0, u, x)=0$ whenever $(0, u, x) \in \Omega$ (notation of (3)), and so

$$
\text { i) } Q^{\prime}(s u+x)=s^{2} \mu(s, u, x) \text {, ii) } \mu(0, u, 0)=(u, u)
$$

for $\mu: \Omega \rightarrow \mathbb{R}$ with $\mu(s, u, x)=\int_{0}^{1}(1-t) \beta_{s s}(t s, u, x) d t$ and all $(s, u, x) \in \Omega$. In fact, (3,ii) yields (9,i), and (9, ii) follows as $2 \mu(0, u, 0)=\partial d Q_{0}^{\prime}(u, u)$, cf. (7).

According to Remark 6.3, the points $u \in \Sigma$ such that $(u, u)=0$ form a (possibly disconnected) codimension-one submanifold $\Pi$ of $\Sigma$, and the function $\Sigma \ni u \mapsto(u, u)$ has a nonzero differential at every point of $\Pi$. For $\Omega_{0}=(-\varepsilon, \varepsilon) \times \Pi \times K$, (9),ii) and compactness of $\Pi$ allow us to choose $\varepsilon$ and $K$ small enough so as to ensure that 
$\mu: \Omega \rightarrow \mathbb{R}$ has a nonzero differential at every point of the codimension-one submanifold $\Omega_{0}$ of $\Omega$. Also, by (9.i), $\mu=0$ on $\Omega_{0}$, since the equality $Q(s u+x)=s^{2}(u, u)$ (see Remark 7.3) gives $\Omega_{0} \subseteq Y \subseteq Y^{\prime}$. Lemma 2.1(b) now guarantees, for smaller $\varepsilon$ and $K$, the existence of an open subset $U$ of $\Sigma$ such that $\Pi \subseteq U$ and $\mu \neq 0$ everywhere in $(-\varepsilon, \varepsilon) \times[U \backslash \Pi] \times K$. Since, in addition, (9),ii) yields $\mu \neq 0$ at all points of the compact set $\{0\} \times[\Sigma \backslash U] \times\{0\}$, making $\varepsilon$ and $K$ even smaller we obtain $\mu \neq 0$ everywhere in $(-\varepsilon, \varepsilon) \times[\Sigma \backslash \Pi] \times K=\Omega \backslash \Omega_{0}$. In view of (9,i), this proves the lemma, with $U^{\prime}=B \oplus K$ for the current choices of $\varepsilon$ and $K$.

The following result is a key technical ingredient for the proof of Theorem C. For the definition of $\mathbb{L}_{z}\left(Z \backslash \phi^{-1}(0), K\right)$, see Section 5.

Theorem 7.5. Given a submanifold $K$ of a manifold $N$, a point $z \in K$, a vector space $\mathcal{T}$ containing $T_{z} N$ as a subspace, a symmetric bilinear form $\langle$,$\rangle in \mathcal{T}$, a vector-valued function $f: N \rightarrow \mathcal{T}$, and a function $\phi: N \rightarrow \mathbb{R}$, for which $d \phi_{z} \neq 0$ and $P=\phi^{-1}(0)$ is a codimension-one submanifold of $N$ such that $K \subseteq Y \subseteq P$ with some quadric $Y$ at $z$ in the manifold $P$ modelled on the restriction of $\langle$,$\rangle to the subspace H=T_{z} P$, let $\partial d f_{z}$ denote the Hessian of $f$ at $z$. In addition, suppose that

(a) the restriction of $\langle$,$\rangle to T_{z} N$ is nonzero,

(b) $V=T_{z} K$ is the nullspace of the restriction of $\langle$,$\rangle to H=T_{z} P$,

(c) $d f=0$ at all points of $K$, and $Y \subseteq Z$, where $Z \subseteq N$ is the zero set of $f$,

(d) $\left\langle w, \partial d f_{z}\right\rangle=d \phi_{z} \otimes\langle w, \cdot\rangle+\langle w, \cdot\rangle \otimes d \phi_{z}-\left[d \phi_{z}(w)\right]\langle$,$\rangle for every w \in T_{z} N$.

Then

(i) $Z \cap P \cap \Omega \subseteq Y$ for some neighborhood $\Omega$ of $z$ in $N$,

(ii) no element of $\mathbb{L}_{z}\left(Z \backslash \phi^{-1}(0), K\right)$ is contained in $(H \backslash V) \cup\{0\}$.

\section{Proof of Theorem 7.5}

In view of (a), both $\langle$,$\rangle and d \phi_{z}$ are nonzero on $T_{z} N$. Let us fix $w \in T_{z} N$ such that $d \phi_{z}(w) \neq 0 \neq\langle w, w\rangle$. The Hessian $\partial d Q_{z}$ of the function $Q: N \rightarrow \mathbb{R}$ with $Q(y)=\langle w, f(y)\rangle$ obviously equals the right-hand side in $(\mathrm{d})$. Thus, if we set $\xi=d \phi_{z}$,

$$
\begin{array}{ll}
\partial d Q_{z}(w, w)=[\xi(w)]\langle w, w\rangle \neq 0 & \text { and, for } u \in H=T_{z} P=\operatorname{Ker} \xi, \\
\partial d Q_{z}(u, u)=-[\xi(w)]\langle u, u\rangle, & \partial d Q_{z}(w, u)=0 .
\end{array}
$$

\subsection{Assertion (i)}

By (b) and (10), the assumptions of Lemma 7.1 hold for $P$ (rather than $N$ ), our $z$, the restriction of $Q$ to $P$, and $K$. Therefore, in view of Lemma $\mathbf{7 . 2}, Y^{\prime}=P \cap Q^{-1}(0)$ is a quadric at $z$ in $P$ modelled on the restriction to $T_{z} P$ of $\partial d Q_{z}$ or, equivalently, of $\langle$,$\rangle (cf. (10) ). Lemma 7.4$ thus applies to $Y^{\prime}$ and the quadric $Y$ in the statement of Theorem 7.5, as the hypotheses $Y \subseteq P$ and $Y \subseteq Z$ in Theorem 7.5, combined with the relation $Z \subseteq Q^{-1}(0)$ (obvious from the definitions of $Z$ and $Q$ ), give $Y \subseteq Z \cap P \subseteq Y^{\prime}$. 
In view of Lemma 7.4, the latter inclusions turn into equalities if one replaces the sets involved by their intersections with a suitable neighborhood $\Omega$ of $z$ in $N$. This not only yields the conclusion $Z \cap P \cap \Omega \subseteq Y$ claimed in (i), but also shows that

$$
f=0 \text { at all points of } P \cap Q^{-1}(0) \text { sufficiently close to } z \text {. }
$$

The remainder of this section is devoted to proving assertion (ii).

\subsection{Identifications and decompositions}

In view of (b) and (10), the hypotheses of Lemma 7.1 are also satisfied by our $N, z, Q$ and $K$. Replacing $N$ by a neighborhood of $z$ in $N$, we may thus use Lemma 7.1 to identify $N$ with a neighborhood of 0 in the vector space $T_{z} N$, and $z$ with 0 , in such a way that $Q$ becomes the quadratic function of the symmetric bilinear form $()=,\partial d Q_{z}$ on $T_{z} N$, and $K$ is the intersection of $N$ with the nullspace of $($,$) . We also decompose$ $T_{z} N$ into a direct sum $W \oplus V$ as in Remark [7.3, choosing $W$ so that $w \in W$. Thus, $V$ is the nullspace of $($,$) . As a result, we obtain three ($,$) -orthogonal decompositions:$

$$
T_{z} N=W \oplus V, \quad W=\mathbb{R} w \oplus H^{\prime}, \quad H=H^{\prime} \oplus V
$$

where $H^{\prime}=w^{\perp} \cap W$, with ()$^{\perp}$ standing for the $($,$) -orthogonal complement in T_{z} N$.

As $V$ is the nullspace of $($,$) , we have K=V \cap N$. Replacing $N$ and $K$ with smaller neighborhoods of 0 in $T_{z} N$ and $V$, we thus get $N=B \oplus K$, meaning that $N=\{y+x: y \in B, x \in K\}$, where $B \subseteq W$ is the open ||-ball in $W$ of some radius $\varepsilon>0$, centered at 0 , for a fixed Euclidean norm || in $T_{z} N$. Summarizing, we have

$$
H^{\prime}=H \cap W, \quad H^{\prime} \subseteq H=T_{z} P=\operatorname{Ker} \xi=w^{\perp}, \quad V=T_{z} K .
$$

As $()=,\partial d Q_{z}$ satisfies (10), it follows from (b) and (12) that

a) the restriction of $($,$) to H^{\prime}$ is nondegenerate,

b) if $($,$) is positive or negative definite on H^{\prime}$, so must be $\langle$,$\rangle ,$

(14),b) being obvious since $($,$) restricted to H^{\prime}$ is, by (10), a nonzero multiple of $\langle$,$\rangle .$

We use the symbol $\Sigma$ for the ||-unit sphere around 0 in $W$.

\subsection{Factorizations of $F, \phi, Q$, and a description of $\mathbb{L}_{z}\left(Z \backslash \phi^{-1}(0), K\right)$}

From now on $(s, u, x)$ denotes a generic element of the open set $\Omega=(-\varepsilon, \varepsilon) \times \Sigma \times K$ in $\mathbb{R} \times \Sigma \times V$. We define a $C^{\infty}$ function $\beta: \Omega \rightarrow T_{z} N$ by $\beta(s, u, x)=f(s u+x)$. As $f$ and $d f$ vanish on $V \cap N=K \subseteq Z$, we have $\beta(0, u, x)=f(0, x)=0$ as well as $\beta_{s}(0, u, x)=d f_{x}(u)=0$ whenever $(0, u, x) \in \Omega$ (notation of (3) ). Similarly, the function $\gamma(s, u, x)=\phi(s u+x)$ vanishes when $s=0$. Thus, $\beta(s, u, x)$ is smoothly divisible by $s^{2}$, and $\gamma(s, u, x)$ by $s$. Explicitly, according to (3),

$$
\begin{aligned}
& f(s u+x)=s^{2} F(s, u, x) \quad \text { with } F(s, u, x)=\int_{0}^{1}(1-t) \beta_{s s}(t s, u, x) d t, \\
& \phi(s u+x)=s \Phi(s, u, x), \quad \text { where } \Phi(s, u, x)=\int_{0}^{1} \gamma_{s}(t s, u, x) d t \\
& Q(s u+x)=s^{2}(u, u)=s^{2}\langle w, F(s, u, x)\rangle .
\end{aligned}
$$


By (15), a vector spanning a line $L \in \mathbb{L}_{z}\left(Z \backslash \phi^{-1}(0), K\right)$ is, up to a factor, the limit of a sequence $v_{j} /\left|v_{j}\right|, j=1,2, \ldots$, where $v_{j}=s_{j} u_{j}+x_{j}-y_{j}$ with $\left(s_{j}, u_{j}, x_{j}\right) \in \Omega$ and $y_{j} \in K$ such that $\left(s_{j}, x_{j}, y_{j}\right) \rightarrow(0,0,0)$, as well as

$$
\Phi\left(s_{j}, u_{j}, x_{j}\right) \neq 0=F\left(s_{j}, u_{j}, x_{j}\right)=\left(u_{j}, u_{j}\right) \quad \text { for all } j \geq 1 .
$$

Passing to a subsequence, we may further assume that $u_{j} \rightarrow u$ for some $u \in \Sigma$, while $s_{j} u_{j} /\left|v_{j}\right| \rightarrow c u$ and $\left(x_{j}-y_{j}\right) /\left|v_{j}\right| \rightarrow x$ for some $c \in \mathbb{R}$ and $x \in V$. Thus, since $F$ is continuous, $F(0, u, 0)=(u, u)=0$. Also, $L=\mathbb{R}(c u+x)$, so that, by (13)),

$$
\text { if } \xi(u) \neq 0 \text {, then } L \text { is not contained in }(H \backslash V) \cup\{0\} \text {. }
$$

\subsection{Values on $\{0\} \times \Sigma \times\{0\}$, where $\Sigma$ is the $\mid$-unit sphere in $W$}

For $\xi=d \phi_{z}$ and any $u \in \Sigma$, the definitions of $\beta$ and $\gamma$ along with (7) and (15) give

$$
\text { i) } 2 F(0, u, 0)=\partial d f_{z}(u, u), \quad \text { ii) } \Phi(0, u, 0)=\xi(u) \text {. }
$$

Consequently, using (d) we see that, if $u \in \Sigma$ and $u^{\prime} \in T_{u} \Sigma$, while $w^{\prime} \in T_{z} N$,

$$
\begin{aligned}
& \text { a) } 2\left\langle w^{\prime}, F(0, u, 0)\right\rangle=2[\xi(u)]\left\langle w^{\prime}, u\right\rangle-\left[\xi\left(w^{\prime}\right)\right]\langle u, u\rangle, \\
& \text { b) }\left\langle w^{\prime}, d F_{(0, u, 0)} u^{\prime}\right\rangle=[\xi(u)]\left\langle w^{\prime}, u^{\prime}\right\rangle+\left[\xi\left(u^{\prime}\right)\right]\left\langle w^{\prime}, u\right\rangle-\left[\xi\left(w^{\prime}\right)\right]\left\langle u, u^{\prime}\right\rangle,
\end{aligned}
$$

with $u^{\prime}$ on the left-hand side standing for the vector $\left(0, u^{\prime}, 0\right)$ tangent to $\{0\} \times \Sigma \times\{0\}$ at $(0, u, 0)$.

In the remainder of the proof, $(-\varepsilon, \varepsilon)$ and $K$ will repeatedly be replaced with smaller neighborhoods of 0 in $\mathbb{R}$ and $V$, as needed for the argument.

\subsection{Case A: (, ) is semidefinite on $H$}

By (14,a), (, ) restricted to $H^{\prime}$ is positive or negative definite. Furthermore,

$$
\xi(u) \neq 0 \text { for every } u \in \Sigma \text { such that } F(0, u, 0)=0,
$$

where $\Sigma \subseteq W$ is the || -unit sphere, $\xi=d \phi_{z}$ and $F$ is given by (15). In fact, suppose that $u \in \Sigma$ and $\xi(u)=0$. Since $\Sigma \subseteq W$, (13) then gives $u \in H^{\prime}$, and so $\langle u, u\rangle \neq 0$ in view of (14, b). Thus, $F(0, u, 0) \neq 0$, as one sees evaluating (19,a) for $w^{\prime}$ equal to the vector $w \in W$ with $\xi(w) \neq 0$ which appears in (10).

Assertion (ii) now follows: for any $\left(s_{j}, u_{j}, x_{j}\right), y_{j}, c, u$ and $x$ with the properties listed in the lines following (15), including $F(0, u, 0)=0$, (20) and (17) yield (ii).

\subsection{Case B: (, ) is not semidefinite on $H$}

This time, (, ) restricted to $H^{\prime}$ is nondegenerate and indefinite, cf. (14.a) and (12). As before, $\Sigma$ denotes the || -unit sphere in $W$, and $\xi=d \phi_{z}$. In view of Remark 6.3, the condition $(u, u)=0$ imposed on $u \in \Sigma$ defines a (possibly disconnected) codimensionone submanifold $\Pi$ of $\Sigma$, containing the subset $\Lambda=\Pi \cap H^{\prime}=\Pi \cap \operatorname{Ker} \xi$ (cf. (13)).

The set $\Lambda$, nonempty due to indefiniteness of $($,$) on H^{\prime}$, contains no critical points

of the restriction $\xi: \Pi \rightarrow \mathbb{R}$. In fact, let $u \in \Lambda$, so that $(u, u)=0$ and $u \in \Sigma^{\prime}$, where 
$\Sigma^{\prime}=\Sigma \cap H^{\prime}$ is the $\mid$-unit sphere in $H^{\prime}$. Remark 6.3 applied to $\Sigma^{\prime}$ shows that the functional $(u, \cdot)$ is nonzero on $T_{u} \Sigma^{\prime}=T_{u} \Sigma \cap \operatorname{Ker} \xi$ (cf. (13)), and $\xi$ is nonzero on $T_{u} \Sigma$ (as $T_{u} \Sigma^{\prime}=T_{u} \Sigma \cap \operatorname{Ker} \xi$ is a proper subspace of $T_{u} \Sigma$ ). The restrictions of the functionals $(u, \cdot)$ and $\xi$ to $T_{u} \Sigma$ are thus linearly independent. Consequently, $\xi$ is nonzero on $T_{u} \Sigma \cap \operatorname{Ker}(u, \cdot)=T_{u} \Pi$, as required.

In terms of $\Omega_{0}=(-\varepsilon, \varepsilon) \times \Pi \times K$, these conclusions and (18,ii) imply that the nonempty set $\Lambda^{\prime}=\{0\} \times \Lambda \times\{0\}$ consists precisely of all zeros of the function $\Phi: \Omega \rightarrow \mathbb{R}$ given by (15) which lie in the submanifold $\{0\} \times \Pi \times\{0\}$ of $\Omega_{0}$, and that the restriction of $\Phi$ to $\{0\} \times \Pi \times\{0\}$ has a nonzero differential at every point of $\Lambda^{\prime}$. As $\Pi$ is compact, choosing smaller $\varepsilon$ and $K$ we can ensure that 0 is a regular value of the restriction $\Phi: \Omega_{0} \rightarrow \mathbb{R}$. In addition, the submersion $s: \Omega_{0} \rightarrow(-\varepsilon, \varepsilon)$, given by $(s, u, x) \mapsto s$, is constant on $\{0\} \times \Pi \times\{0\}$, and so $\Phi$ and $s$, as functions on $\Omega_{0}$, have linearly independent differentials at each point of $\Lambda^{\prime}$. For even smaller $\varepsilon$ and $K$, we thus have $d \Phi \wedge d s \neq 0$ everywhere in $\Omega_{0} \cap \Phi^{-1}(0)$. Thus, $\Omega_{0} \cap \Phi^{-1}(0)$ is a (possibly disconnected) codimension-one submanifold of $\Omega_{0}$, and the additional condition $s=0$ defines a further codimension-one submanifold of $\Omega_{0} \cap \Phi^{-1}(0)$ (so that, in particular, $s \neq 0$ on a dense subset of $\left.\Omega_{0} \cap \Phi^{-1}(0)\right)$. Next, for $F$ given by (15),

(*) $F: \Omega_{0} \rightarrow \mathcal{T}$ has the value 0 and nonzero differential at every point $(s, u, x)$ of the codimension-one submanifold $\Omega_{0} \cap \Phi^{-1}(0)$ of $\Omega_{0}$, containing $\Lambda^{\prime}=\{0\} \times \Lambda \times\{0\}$.

\subsection{Justification of $(*)$}

As $P=\phi^{-1}(0)$, (11) and (15) along with the definitions of $\Pi$ and $\Omega_{0}$ give $F=0$ on $\Omega_{0} \cap \Phi^{-1}(0)$, while $\Lambda^{\prime} \subseteq \Omega_{0} \cap \Phi^{-1}(0)$ due to the definition of $\Lambda$ and (18)ii). Note that the conclusion here is $F=0$, rather than just $s^{2} F=0$, since, as mentioned above, the subset $s \neq 0$ is dense in $\Omega_{0} \cap \Phi^{-1}(0)$.

Since $\Pi$ is compact and we are free to make $\varepsilon$ and $K$ smaller, $(*)$ will follow if we prove it just for $(s, u, x)=(0, u, 0)$ with $u \in \Lambda$, while restricting $F$ further, to the submanifold $\{0\} \times \Pi \times\{0\}$ of $\Omega_{0}$. (As we saw, $\Lambda^{\prime}$ is the intersection of $\Omega_{0} \cap \Phi^{-1}(0)$ with $\{0\} \times \Sigma \times\{0\}$.) We thus only need to show that, whenever $u \in \Sigma$ and $(u, u)=\xi(u)=0$, the right-hand side of (19, b) is nonzero for suitable $w^{\prime} \in T_{z} N$ and $u^{\prime} \in W$ with $\left(u, u^{\prime}\right)=0$. Let us thus set $u^{\prime}=w$, so that $\left(u, u^{\prime}\right)=0 \neq \xi\left(u^{\prime}\right)$ in view of (10). Also, as $($,$) is nondegenerate on H^{\prime}$ (see (14.a)), we may choose $w^{\prime} \in H^{\prime}$ with $\left(w^{\prime}, u\right) \neq 0$ (that is, by (10), $\left\langle w^{\prime}, u\right\rangle \neq 0$ ), and hence $\xi\left(w^{\prime}\right)=0$ by (13). Then $\left[\xi\left(u^{\prime}\right)\right]\left\langle w^{\prime}, u\right\rangle \neq 0$, and the other two terms on the right-hand side of (19, b) vanish.

\subsection{The final step}

We now conclude the argument in Case B. First, by $(*)$ and Lemma 2.1(b), $F \neq 0$ everywhere in $U \backslash \Phi^{-1}(0)$ for some open set $U \backslash \Omega_{0}$ containing $\Omega_{0} \cap \Phi^{-1}(0)$.

Let us now fix any $\left(s_{j}, u_{j}, x_{j}\right), y_{j}, c, u$ and $x$ satisfying the conditions in the lines following (15), which include (16) and $F(0, u, 0)=(u, u)=0$. We then also have 
$\xi(u) \neq 0$ (which, in view of (17), yields (ii)). To see this, suppose that, on the contrary, $\xi(u)=0$. Thus, $(0, u, 0) \in \Lambda^{\prime} \subseteq \Omega_{0} \cap \Phi^{-1}(0)$ (see (*)) and, by (16)), $\left(s_{j}, u_{j}, x_{j}\right) \in \Omega_{0} \backslash \Phi^{-1}(0)$. Also, $\left(s_{j}, u_{j}, x_{j}\right) \rightarrow(0, u, 0)$, so that, if $j$ is sufficiently large, $\left(s_{j}, u_{j}, x_{j}\right)$ must lie in $U \backslash \Phi^{-1}(0)$. Hence, according to the last paragraph, $F\left(s_{j}, u_{j}, x_{j}\right) \neq 0$ for large $j$, which contradicts (16), completing the proof.

\section{Conformal vector fields}

The symbol $\nabla$ always stands both for the Levi-Civita connection and the gradient operator of a given pseudo-Riemannian manifold $(M, g)$. If $v$ is a vector field on $M$, denoting by $A=\nabla v-[\nabla v]^{*}$ twice the skew-adjoint part of $\nabla v$, with $\nabla v: T M \rightarrow T M$ as in the lines following (2), we can rewrite condition (11) as

$$
2 \nabla v=A+\phi \mathrm{Id} .
$$

We then obviously have $e^{\tau} £_{v}\left(e^{-\tau} g\right)=£_{v} g-[(d \tau)(v)] g$ for any function $\tau: M \rightarrow \mathbb{R}$, and so, under the assumption (1), the condition $(d \tau)(v)=\phi$ is necessary and sufficient in order that $v$ be a Killing field for the metric $e^{-\tau} g$ conformal to $g$. At points where $v$ is nonzero, $\tau$ with $(d \tau)(v)=\phi$ always exists locally, due to solvability of ordinary differential equations. Thus, such points are never essential (cf. the Introduction).

Lemma 9.1. Let $z \in M$ be a zero of a conformal vector field $v$ on a pseudo-Riemannian manifold $(M, g)$, and let $\phi$ be the function in (11).

(a) If $\phi(z) \neq 0$, then Ker $\nabla v_{z}$ is a null subspace of $T_{z} M$.

(b) If $\phi(z)=0$, then Ker $\nabla v_{z}$ has even codimension in $T_{z} M$, and its orthogonal complement is the image $\nabla v_{z}\left(T_{z} M\right)$.

Proof. This is immediate since, in view of (21), Ker $\nabla v_{z} \backslash\{0\}$ consists of eigenvectors of the skew-adjoint operator $A_{z}: T_{z} M \rightarrow T_{z} M$ for the eigenvalue $-\phi(z)$.

It is well-known [7, 8, 12, 6] that (1) implies further differential equations. In dimensions $n \geq 3$, this allows us to identify conformal vector fields on $(M, g)$ with parallel sections of a certain vector bundle over $M$, carrying a natural connection; consequently, the dimension of the space of conformal fields on $(M, g)$ cannot exceed $(n+1)(n+2) / 2$. Specifically, if $v$ satisfies (1) on $(M, g)$, and $\operatorname{dim} M=n \geq 2$, then

a) $2 \nabla_{u} \nabla v=2 R(v \wedge u)+d \phi \otimes u-g(u, \cdot) \otimes \nabla \phi+[(d \phi)(u)] \mathrm{Id}$,

b) $(1-n / 2)[\nabla d \phi](u, u)=S\left(u, \nabla_{u} v\right)+S\left(u, \nabla_{u} v\right)+\left[\nabla_{v} S\right](u, u)$

for all vector fields $u$, where both sides in (22, a) are bundle morphisms $T M \rightarrow T M$, the symbol $R$ stands for the curvature tensor, with the sign convention $R(w \wedge u) u^{\prime}=$ $\nabla_{u} \nabla_{w} u^{\prime}-\nabla_{w} \nabla_{u} u^{\prime}+\nabla_{[w, u]} u^{\prime}$ for vector fields $w, u, u^{\prime}$, and $S=\operatorname{Ric}-(2 n-2)^{-1} \sigma g$ is the Schouten tensor, with $\sigma$ denoting the scalar curvature. In coordinates, $2 v^{l}{ }_{k j}=$ $2 R_{p j k} v^{p}+\phi_{, k} \delta_{j}^{l}-\phi^{, l} g_{j k}+\phi_{, j} \delta_{k}^{l}$ and $(1-n / 2) \phi_{, j k}=S_{j p} v^{p}{ }_{, k}+S_{k p} v^{p}{ }_{, j}+S_{j k, p} v^{p}$. In fact, the coordinate version of $(22, \mathrm{a})$ follows from the more general fact that, given a 1 -form $\xi$ on a manifold with a torsionfree connection, setting $a_{j k}=\xi_{k, j}+\xi_{j, k}$, one obtains 
$\xi_{l, k j}=R_{l k j}^{p} \xi_{p}+\left(a_{l j, k}+a_{l k, j}-a_{k j, l}\right) / 2$, in view of the Ricci and Bianchi identities, cf. [9, the bottom of p. 572].

Equality (22, b) can be justified as follows (with (1) and (22) always meaning the coordinate versions). First, due to the second Bianchi identity, $R_{p j k}{ }^{l}{ }_{l}=R_{k p, j}-R_{k j, p}$, while the Bochner formula (contracted Ricci identity) gives $v_{, k l}^{l}=R_{k p} v^{p}+n \phi_{, k} / 2$ (as $\left.v^{p}{ }_{, p}=n \phi / 2\right)$, and hence $v^{l}{ }_{, k l j}=R_{k p, j} v^{p}+R_{k p} v^{p}{ }_{, j}+n \phi_{, j k} / 2$. Subtracting the Ricci identity $v^{l}{ }_{, k j l}-v^{l}{ }_{, k l j}=R_{p j k} v^{p}{ }_{, l}+R_{j p} v^{p}{ }_{, k}$ from $1 / 2$ times the formula obtained by applying $\nabla_{l}$ to (22, a), and using the above expressions for $R_{p j k}{ }_{, l}$ and $v^{l}{ }_{, k l j}$, we see that $(1-n / 2) \phi_{, j k}=R_{j p} v^{p}{ }_{, k}+R_{k p} v^{p}{ }_{j}+R_{j k, p} v^{p}+\phi_{, l}{ }^{l} g_{j k} / 2$. Now (22. b) easily follows from (1) since $R_{j k}=S_{j k}+(2 n-2)^{-1} \sigma g_{j k}$ and $(1-n) \phi_{, k}{ }^{k}=\sigma \phi+(d \sigma)(v)$. The last relation is another general consequence of (1): $\phi_{, k}{ }^{k}=\left(\phi g_{j k}\right)^{j k}=\left(v_{j, k}+v_{k, j}\right)^{, j k}=$ $2 v^{l}{ }_{, k l}^{k}=2\left(R_{k p} v^{p}\right)^{, k}+n \phi_{, k}{ }^{k}$ in view of the equality $v^{j, k}{ }_{j k}=v^{j, k}{ }_{k j}$ (immediate from the Ricci identity) and the Bochner formula just mentioned; on the other hand, $2\left(R_{k p} v^{p}\right)^{, k}=R^{j k}\left(v_{j, k}+v_{k, j}\right)+2 R_{k p}{ }^{k} v^{p}=\sigma \phi+(d \sigma)(v)$, as the Bianchi identity for the Ricci tensor gives $2 R_{k p}{ }^{k}=\sigma_{, p}$.

\section{The case of pseudo-Euclidean spaces}

Let $V$ be an $n$-dimensional pseudo-Euclidean space with the inner product $\langle$,$\rangle . For$ any $w, u \in V$, any skew-adjoint endomorphism $B: V \rightarrow V$, and $c \in \mathbb{R}$, the formula

$$
v_{x}=w+B x+c x+2\langle u, x\rangle x-\langle x, x\rangle u
$$

is easily seen to define a conformal vector field $v$ on $(V, g)$, where $g$ is the constant flat metric correspoding to $\langle$,$\rangle . If n \geq 3$, the resulting vector space of conformal fields has the maximum possible dimension $(n+1)(n+2) / 2$ (See the lines preceding (22).) Thus, (23) describes all conformal fields on any open submanifold of $(V, g)$.

Defining $v$ by (23) with $w=0$ and $c=0$, we see that $v=0$ everywhere in the set $\Pi=\{x \in \operatorname{Ker} B:\langle u, x\rangle=\langle x, x\rangle=0\}$. If, in addition, $u$ does not lie in the image $B(V)$, then all zeros $x$ of $v$ sufficiently close to 0 lie in $\Pi$. In fact, as $0=\left\langle v_{x}, x\right\rangle=\langle u, x\rangle\langle x, x\rangle$, it follows that $\langle x, x\rangle=0$, or else the equality $0=v_{x}=B x+2\langle u, x\rangle x-\langle x, x\rangle u$ with $\langle u, x\rangle=0$ would give $u \in B(V)$. Thus, $0=v_{x}=B x+2\langle u, x\rangle x$. Such $x$ which also have the property that $\langle u, x\rangle \neq 0$ cannot be arbitrarily close to 0 , since they all lie in hyperplanes given by $2\langle u, x\rangle=-b$, where $b$ ranges over nonzero eigenvalues of $B$. Consequently, $\langle u, x\rangle=\langle x, x\rangle=0$ for all zeros of $v$ near 0 , and then also $0=v_{x}=B x$.

EXAMPLE 10.1. For $v$ as in the last paragraph, let $Z^{\prime}$ be the connected component of the zero set of $v$ containing $\Pi$. If $B$ and $u$ are chosen so that the restriction of $\langle$, to $u^{\perp} \cap \operatorname{Ker} B$ is not semidefinite, $Z^{\prime}$ will have a singularity at 0 (Remark 6.2(a)). On the other hand, semidefiniteness of $\langle$,$\rangle on u^{\perp} \cap \operatorname{Ker} B$ implies that $\Pi$ is a submanifold of $V$ (see Remark 6.1), while, if $\langle$,$\rangle is indefinite, one can also choose such B$ and $u$ for which, in addition, $\operatorname{dim} \Pi \geq 1$ and $\operatorname{dim} V-\operatorname{dim} \Pi$ is odd. 
EXAMPLE 10.2. In $\mathbf{R}^{2}$ with the Cartesian coordinates $x^{j}$, let $g$ be the flat metric given by $g_{12}=g_{21}=1$ and $g_{11}=g_{22}=0$. The conformal vector fields $v$ for $g$ are obviously characterized by the partial derivative conditions $\partial_{1} v_{1}=\partial_{2} v_{2}=0$, that is, $\partial_{1} v^{2}=\partial_{2} v^{1}=0$. Hence $v^{1}$ may be any function of $x^{1}$ and $v^{2}$ any function of $x^{2}$. Thus, the zero set of $v$ can have the form $\Xi \times \Xi^{\prime}$, with any closed sets $\Xi, \Xi^{\prime} \subseteq \mathbb{R}$.

\section{Intermediate submanifolds}

The proof of Theorem C under the assumption (2. b), given in Section 16, uses a submanifold $N$ containing all zeros of a given conformal vector field that lie near a prescribed zero $z$, and having the tangent space $\operatorname{Ker} \nabla v_{z}$ at $z$. According to Example 3.1, such $N$ always exists. For easy reference, this fact and some properties of $N$ are gathered in the following lemma. Radial limit directions of a set are defined at the end of Section 5 ,

Lemma 11.1. Given a conformal vector field $v$ on a pseudo-Riemannian manifold $(M, g)$ and a zero $z \in M$ of $v$, there exists a submanifold $N$ of $M$ such that

(i) all zeros of $v$ sufficiently close to $z$ lie in $N$,

(ii) $T_{x} N=\operatorname{Ker} \nabla v_{x}$ at every $x \in N$ at which $v_{x}=0$ and $\operatorname{rank} \nabla v_{x}=\operatorname{rank} \nabla v_{z}$,

(iii) all radial limit directions of the zero set of $v$ at $z$ lie in $T_{z} N=\operatorname{Ker} \nabla v_{z}$,

(iv) if $\operatorname{rank} \nabla v_{x}=\operatorname{rank} \nabla v_{z}$ at a point $x \in N$ at which $v_{x}=0$, and $w$ is a vector field on $M$ with $w_{x} \in \operatorname{Ker} \nabla v_{x}$, while $\phi(x)=0$, for $\phi$ in (1), then the function $Q=2 g(w, v)$ restricted to $N$ has a critical point at $x$, and its Hessian $\partial d Q_{x}$

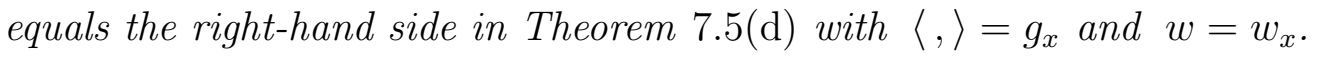

Proof. For $N$ constructed in Example 3.1, with $\mathcal{E}=T M$ and $\psi=v$, one clearly has (i) and (ii), while (iii) follows from Remark 5.1(i). Finally, in (iv), the condition $w_{x} \in \operatorname{Ker} \nabla v_{x}$ implies, by Lemma 9.1(b), that the value $\xi_{x}$ of the 1 -form $\xi=2 g(w, \cdot)$ vanishes on the image $\nabla v_{x}\left(T_{x} M\right)$. Now (iv) is obvious from the Hessian formula in Remark 3.3(ii), combined with the expression for the second covariant derivative of $v$ at $x$ provided by (22, a), in which the curvature term vanishes since $v_{x}=0$.

\section{Conformal fields along geodesics}

Given a conformal vector field $v$ on a pseudo-Riemannian manifold $(M, g)$ of dimension $n \geq 3$, with (1), let us consider a parallel vector field $t \mapsto u(t) \in T_{x(t)} M$ along a geodesic

$t \mapsto x(t)$ of $(M, g)$. Transvecting the coordinate versions of (1) and (22) with $\dot{x}^{j} \dot{x}^{k}$ or, respectively, $\dot{x}^{j} u^{k}$, we obtain

$$
\begin{aligned}
\text { i) } & 2\langle v, \dot{x}\rangle^{\cdot}=\phi\langle\dot{x}, \dot{x}\rangle, \\
\text { ii) } & 2 \nabla_{\dot{x}} \nabla_{u} v=2 R(v \wedge \dot{x}) u+[(d \phi)(u)] \dot{x}+\dot{\phi} u-\langle\dot{x}, u\rangle \nabla \phi, \\
\text { iii) } & (1-n / 2)[(d \phi)(u)]^{\cdot}=S\left(u, \nabla_{\dot{x}} v\right)+S\left(\dot{x}, \nabla_{u} v\right)+\left[\nabla_{v} S\right](u, \dot{x}),
\end{aligned}
$$


where $(,)^{*}=d / d t$, cf. (6) , the symbol $\langle$,$\rangle stands for g$, and the dependence of both sides on $t$ suppressed in the notation: $v=v_{x(t)}, \phi=\phi(x(t))$. With $u=\dot{x}$, (24) gives

$$
\begin{aligned}
& \text { i) } \quad \nabla_{\dot{x}} \nabla_{\dot{x}} v=R(v \wedge \dot{x}) \dot{x}+\dot{\phi} \dot{x}-\langle\dot{x}, \dot{x}\rangle \nabla \phi / 2, \\
& \text { ii) } \quad(1-n / 2) \ddot{\phi}=2 S\left(\dot{x}, \nabla_{\dot{x}} v\right)+\left[\nabla_{v} S\right](\dot{x}, \dot{x}) \text {. }
\end{aligned}
$$

As a consequence of (25) i), condition (1) implies that

$$
\nabla_{\dot{x}} \nabla_{\dot{x}}(v \wedge \dot{x})=[R(v \wedge \dot{x}) \dot{x}] \wedge \dot{x} \quad \text { if } t \mapsto x(t) \text { is a null geodesic. }
$$

If, in addition, $v$ is tangent to a null geodesic $t \mapsto x(t)$, that is, $v_{x(t)}$ is a multiple of $\dot{x}(t)$ for every $t$, then, by (25), i) with $v \wedge \dot{x}=0$ and $\langle\dot{x}, \dot{x}\rangle=0$,

$$
\nabla_{\dot{x}} \nabla_{\dot{x}} v=\dot{\phi} \dot{x} .
$$

REMARK 12.1. If two distinct zeros $z, x$ of a conformal vector field $v$ on a pseudoRiemannian manifold $(M, g)$ are joined by a non-null geodesic segment $\Gamma$ and $\phi$ is the function in (1), then $\phi=0$ somewhere in $\Gamma \backslash\{z, x\}$.

This is clear since, in (24) $\mathrm{i}),\langle v, \dot{x}\rangle=0$ at both ends of the parameter interval.

In the next lemma, $T_{\Gamma} M$ denotes the restriction of $T M$ to the one-dimensional null immersed submanifold $\Gamma$. Thus, $T \Gamma$ and $(T \Gamma)^{\perp}$ are subbundles of $T_{\Gamma} M$, while $g$ obviously induces a (nondegenerate) fibre metric in the quotient bundle $(T \Gamma)^{\perp} /(T \Gamma)$. By $\operatorname{conf}\left[(T \Gamma)^{\perp} /(T \Gamma)\right]$ we denote the vector bundle over $\Gamma$ whose sections are infinitesimal conformal endomorphism of $(T \Gamma)^{\perp} /(T \Gamma)$. (An infinitesimal conformal endomorphism is one with the self-adjoint part equal to a function times Id.)

Lemma 12.2. Let a conformal vector field $v$ on a pseudo-Riemannian manifold $(M, g)$ be tangent to a nontrivial null geodesic $\Gamma$ with a parametrization $[0,1] \ni t \mapsto x(t)$, so that $x(0)=y$ and $\nabla_{\dot{x}} v=\lambda \dot{x}$ at $t=0$, for some $y \in M$ and $\lambda \in \mathbb{R}$.

(a) Along $\Gamma$, we have $\nabla_{\dot{x}} v=[\lambda+\phi-\phi(y)] \dot{x}$, with $\phi$ as in (11).

(b) If $\phi$ is constant along $\Gamma$ and $n=\operatorname{dim} M \geq 2$, then $\nabla v$, restricted to $\Gamma$,

(i) acts on $T \Gamma$ and $\left(T_{\Gamma} M\right) /(T \Gamma)^{\perp}$ as the multiplications by $\lambda$ and $\phi-\lambda$,

(ii) descends to a parallel section of conf $\left[(T \Gamma)^{\perp} /(T \Gamma)\right]$ with the trace $(n-2) \phi / 2$,

(iii) has the same characteristic polynomial at all points of $\Gamma$.

Proof. Integrating (27), we obtain (a), while (b-i) for $T \Gamma$ is obvious from (a) with $\phi=\phi(y)$. Next, in view of (21) with $A^{*}=-A$, the subbundles $T \Gamma$ and $(T \Gamma)^{\perp}$ of $T_{\Gamma} M$ are $\nabla v$-invariant; $\nabla v$-invariance of the latter follows from that of the former, since it is the same as $A$-invariance. Thus, $\nabla v$ descends to an endomorphism of $(T \Gamma)^{\perp} /(T \Gamma)$, which is obviously conformal and has the trace claimed in (b-ii), since, by (11), $\nabla v+[\nabla v]^{*}=\phi$ Id.

Let $t \mapsto u(t)$ be any vector field along $\Gamma$. Using (21) with $A^{*}=-A$ and (a) with $\phi=\phi(y)$, we see that $2\left\langle\dot{x}, \nabla_{u} v\right\rangle=\langle\dot{x}, A u+\phi u\rangle=\langle\phi \dot{x}-A \dot{x}, u\rangle=2\left\langle\phi \dot{x}-\nabla_{\dot{x}} v, u\right\rangle=$ $2\langle\dot{x},(\phi-\lambda) u\rangle$, which proves (b-i) for $\left(T_{\Gamma} M\right) /(T \Gamma)^{\perp}$. Now, if $\nabla_{\dot{x}} u=0$ and $\langle\dot{x}, u\rangle=0$, (24).ii) implies that $2 \nabla_{\dot{x}} \nabla_{u} v=[(d \phi)(u)] \dot{x}$, as our assumptions give $v \wedge \dot{x}=0$ and $\dot{\phi}=0$. Thus, $\nabla_{u} v$ projects onto a parallel section of $(T \Gamma)^{\perp} /(T \Gamma)$, which proves (b-ii). 
Due to $\nabla v$-invariance of the subbundles $T \Gamma,(T \Gamma)^{\perp}$ of $T_{\Gamma} M$ and the inclusion $T \Gamma \subseteq(T \Gamma)^{\perp}$, the characteristic polynomial of $\nabla v$ in $T_{\Gamma} M$ equals the product of the characteristic polynomials of the endomorphisms induced by $\nabla v$ in the three bundles $T \Gamma,(T \Gamma)^{\perp} /(T \Gamma)$ and $\left(T_{\Gamma} M\right) /(T \Gamma)^{\perp}$. By (b-i), the first and last of these are polynomials of degree one with the roots $\lambda$ and $\phi-\lambda$, constant along $\Gamma$, while the second polynomial is constant along $\Gamma$ as a consequence of (b-ii), which completes the proof.

Lemma 12.3. Let there be given a conformal vector field $v$ on a pseudo-Riemannian manifold $(M, g)$ of dimension $n \geq 3$, a point $z \in M$, a nonzero null vector $w \in T_{z} M$, and a nontrivial geodesic segment $\Gamma$ in $(M, g)$ containing $z$ and tangent to $w$ at $z$.

(a) If $v_{z}=0$ and $w \in \operatorname{Ker} \nabla v_{z} \cap \operatorname{Ker} d \phi_{z}$, for the function $\phi$ in (1), then $v=0$ and $\phi=\phi(z)$ at every point of $\Gamma$.

(b) If $v_{z}=0$ and $v_{x}=0$ for some point $x \in \Gamma \backslash\{z\}$ lying in a normal-coordinate neighborhood $U^{\prime}$ of $z$, or $v_{z}$ and $\nabla_{w} v \in T_{z} M$ are both tangent to $\Gamma$ at $z$, then $v$ is tangent to $\Gamma$ at every point of $\Gamma$.

Proof. Let $t \mapsto x(t)$ be a geodesic parametrization of $\Gamma$ with $x(0)=z$. As $\langle\dot{x}, \dot{x}\rangle=0$, (25) constitutes a system of first-order linear homogeneous ordinary differential equations with the unknowns $v, \nabla_{\dot{x}} v$ and $\dot{\phi}$, for which the assumption in (a) amount to choosing the zero initial conditions at $t=0$. The conclusion of (a) is now obvious from uniqueness of solutions. Similarly, if $v$ and $\nabla_{\dot{x}} v$ are both tangent to the geodesic at $t=0$, (26) implies, for the same reason, that $v \wedge \dot{x}=0$ at every $t$, proving (b) in this case. Finally, if $v_{z}=0=v_{x}$ for $x$ as in (b), the local flow of $v$ sends the portion of $\Gamma$ joining $z$ to $x$ into itself (Remark 4.1). Combined with the preceding sentence, this shows that $v$ is tangent to $\Gamma$.

Lemma 12.4. Suppose that $[0,1] \ni t \mapsto x(t)$ is a parametrization of a nontrivial null geodesic segment $\Gamma$ with the endpoints $z=x(0)$ and $y=x(1)$ in a pseudo-Riemannian manifold $(M, g)$, while $v$ is a conformal vector field on $(M, g)$ vanishing at both $z$ and $x$. For $\phi$ and $\dot{\phi}$ as in (11) and (6), one has

(i) if $v$ is tangent to $\Gamma$, then $\nabla_{\dot{x}} v=0$ somewhere in $\Gamma \backslash\{z, y\}$, and

(ii) if $\nabla_{\dot{x}} v=0$ at $z$, then $\phi-\phi(z)$ and $\dot{\phi}$ vanish at some points of $\Gamma \backslash\{z, y\}$.

Proof. By Lemma 12.3(b), $v$ is tangent to $\Gamma$ in case (ii) as well. Since $v_{x(t)}$ is a multiple of $\dot{x}(t)$, it may be viewed as a function $[0,1] \rightarrow \mathbb{R}$, equal to 0 at the endpoints. Its derivative $\nabla_{\dot{x}} v$ therefore vanishes at some $t \in(0,1)$. Under the assumption of (ii), $\nabla_{\dot{x}} v=0$ both at 0 and somewhere in $(0,1)$, so that (27) (for the second derivative $\left.\nabla_{\dot{x}} \nabla_{\dot{x}} v\right)$ and the equality $\nabla_{\dot{x}} v=[\phi-\phi(z)] \dot{x}$, due to Lemma 12.2(a), imply (ii).

\section{The first of the two inclusions}

Assertion (a) in the following lemma implies a part of the conclusion in Theorem C. See Remark 13.2 for details. 
Lemma 13.1. For a conformal vector field $v$ on a pseudo-Riemannian manifold $(M, g)$ of dimension $n \geq 3$, a point $z \in M$ with $v_{z}=0$, and $\phi$ as in (11), let us set $V=H \cap H^{\perp}$ and $E=C \cap H$, where $H=\operatorname{Ker} \nabla v_{z} \cap \operatorname{Ker} d \phi_{z}$ and $C=\left\{u \in T_{z} M: g_{z}(u, u)=0\right\}$ denotes the null cone, so that $V$ is a null vector subspace of $T_{z} M$ contained in the subset $E$. Whenever $U$ is a sufficiently small star-shaped neighborhood of 0 in $T_{z} M$ mapped by $\exp _{z}$ diffeomorphically onto a neighborhood of $z$ in $M$, the image $K=\exp _{z}[V \cap U]$ is a submanifold of $M$, while

(a) $v=0$ and $\phi=\phi(z)$ everywhere in $\exp _{z}[E \cap U]$, and hence everywhere in $K$,

(b) for any $x \in K$, the parallel transport along the geodesic contained in $K$ which joins $z$ to $x$ sends $H$ onto $\mathcal{H}_{x}=\operatorname{Ker} \nabla v_{x} \cap \operatorname{Ker} d \phi_{x}$,

(c) $\operatorname{dim} \mathcal{H}_{x}$ is constant as a function of $x \in K$,

(d) if $\phi(z)=0$, then $\operatorname{rank} \nabla v_{x}$ and $\operatorname{dim} \operatorname{Ker} \nabla v_{x}$ are constant as functions of $x \in K$.

Proof. Lemma 12.3(a) implies (a). Next, if $t \mapsto u(t) \in T_{x(t)} M$ is a parallel vector field along a geodesic $t \mapsto x(t)$ in $K$ with $x(0)=z$ and $u(0) \in H$, the equality $V=H \cap H^{\perp}$ gives $\langle\dot{x}, u\rangle=0$ at $t=0$ and, consequently, for all $t$. (As usual, $\langle$,$\rangle stands for g$.) By (a), $v=\nabla_{\dot{x}} v=0$ and $\dot{\phi}=0$ along the whole geodesic, so that equations (24).ii) - (24,iii) now read $2 \nabla_{\dot{x}} \nabla_{u} v=[(d \phi)(u)] \dot{x}$ and $(1-n / 2)[(d \phi)(u)]^{\circ}=S\left(\dot{x}, \nabla_{u} v\right)$. This is a system of first-order linear homogeneous ordinary differential equations with the unknowns $\nabla_{u} v$ and $(d \phi)(u)$, which equal zero at $t=0$ and, therefore, at every $t$. The parallel transport along the geodesic from $z$ to $x=x(t)$ thus sends $\mathcal{H}_{z}$ into $\mathcal{H}_{x}$. The word 'into' can be replaced with 'onto' if $U$ is small enough. Namely, $\mathcal{H}_{x}$ is the kernel of a linear operator depending continuously on $x \in K$, and so $\operatorname{dim} \mathcal{H}_{x}$ is semicontinuous: $\operatorname{dim} \mathcal{H}_{x} \leq \operatorname{dim} \mathcal{H}_{z}$ for $x$ near $z$ in $K$. However, the "into" conclusion established above gives $\operatorname{dim} \mathcal{H}_{x} \geq \operatorname{dim} \mathcal{H}_{z}$. Now (b) and (c) follow. Finally, $p_{z}-1 \leq p_{x} \leq p_{z}$ for $p_{x}=\operatorname{dim} \operatorname{Ker} \nabla v_{x}$ and all $x \in K$ close to $z$. In fact, $p_{x} \leq p_{z}$ due, again, to semicontinuity, and $p_{z}-1 \leq \operatorname{dim} \mathcal{H}_{z}=\operatorname{dim} \mathcal{H}_{x} \leq p_{x}$ by (c). On the other hand, if $\phi=0$ at $z$, (a) gives $\phi=0$ on $K$. Thus, $n-p_{x}$ is even (Lemma 9.1(b)), which, combined with the inequality $p_{z}-1 \leq p_{x} \leq p_{z}$, yields $(\mathrm{d})$.

REMARK 13.2. Proving Theorem Cl has now been reduced to showing that

$$
Z \cap U^{\prime} \subseteq \exp _{z}[C \cap H \cap U] \text { for sufficiently small } U \text { and } U^{\prime},
$$

since the opposite inclusion is provided by Lemma 13.1(a).

\section{Connecting limits for the zero set}

This section consists of two lemmas needed in the proof of Theorem $\mathrm{C}$. For the definition of $\mathbb{L}_{z}\left(Z \backslash \phi^{-1}(0), K\right)$, see Section 5 ,

Lemma 14.1. For $M, g, v, Z, z, \phi$ and $H$ as in Theorem $\mathbb{C}$, let $V=H \cap H^{\perp}$. If $\phi(z)=0$ and $\nabla \phi_{z} \notin \nabla v_{z}\left(T_{z} M\right)$, while no element of $\mathbb{L}_{z}\left(Z \backslash \phi^{-1}(0), K\right)$ is contained in $(H \backslash V) \cup\{0\}$, then $\phi=0$ at every zero of $v$ sufficiently close to $z$. 
Proof. We fix a normal-coordinate neighborhood $U^{\prime}$ of $z$ which is subconvex (Section 4), a star-shaped neighborhood $U$ of 0 in $T_{z} M$ mapped by $\exp _{z} \operatorname{diffeomorphi-}$ cally onto $U^{\prime}$, and a Riemannian metric $h$ on $U^{\prime}$. By Lemma 13.1(a), $K=\exp _{z}[V \cap U]$ is a submanifold of $U^{\prime}$ contained in both $\phi^{-1}(0)$ and the zero set $Z$ of $v$. In view of the inverse mapping theorem, applied to the $g$-exponential mapping of the $h$-normal bundle of $K$ in $U^{\prime}$, by making $U^{\prime}$ smaller we can also ensure that every $y \in U^{\prime} \backslash K$ is joined to some point $p_{y} \in K$ by a nontrivial $g$-geodesic segment $\Gamma_{y}$ contained in a normal-coordinate neighborhood of $p_{y}$ and $h$-normal to $K$ at $p_{y}$.

Furthermore, $\phi \neq 0$ everywhere in $\Gamma_{y} \backslash\left\{y, p_{y}\right\}$, for all $y \in\left(Z \cap U^{\prime}\right) \backslash \phi^{-1}(0)$, as long as $U^{\prime}$ is sufficiently small. Namely, if this were not the case, there would exist a sequence of points $y \in\left(Z \cap U^{\prime}\right) \backslash \phi^{-1}(0)$ converging to $z$ with $\phi=0$ at some interior point of each $\Gamma_{y}$. As $\phi=0$ at the endpoint $p_{y}$ due to the inclusion $K \subseteq \phi^{-1}(0)$, the tangent direction of each $\Gamma_{y}$ at some other interior point would thus be contained in Ker $d \phi$. In view of Lemma 5.2 and Remark 5.1(ii), a subsequence of such a sequence of interior tangent directions would converge to an element $L$ of $\mathbb{L}_{z}\left(Z \backslash \phi^{-1}(0), K\right)$ contained in both $\operatorname{Ker} \nabla v_{z}$ and $\operatorname{Ker} d \phi_{z}$, so that $L \subseteq H=\operatorname{Ker} \nabla v_{z} \cap \operatorname{Ker} d \phi_{z}$. By Lemma 5.2, the tangent direction of $\Gamma_{y}$ at the endpoint $p_{y}$ would tend to $L$ as well, so that $L$ would be $h$-normal to $K$ at $z$, and hence not contained in $V=T_{z} K$, contrary to our assumption.

We now show that $\left(Z \cap U^{\prime}\right) \backslash \phi^{-1}(0)=\varnothing$ for small enough $U^{\prime}$, as required. In fact, otherwise we might fix a sequence of distinct points $y \in\left(Z \cap U^{\prime}\right) \backslash \phi^{-1}(0)$ converging to $z$. In view of the last paragraph and Remark 12.1, each of the geodesic segments $\Gamma_{y}$ is null. Since the null geodesic segment $\Gamma_{y}$ lies in a normal-coordinate neighborhood of $p_{y}$, while $v=0$ at both $p_{y}$ and $y$, Lemma 12.3(b) implies that $v$, and hence $\nabla_{\dot{x}} v$, is tangent to $\Gamma_{y}$. In terms of a geodesic parametrization $[0,1] \ni t \mapsto x(t)$ of $\Gamma_{y}$ such that $x(0)=y$, we thus have, by Lemma 12.2(a), $\nabla_{\dot{x}} v=[\lambda+\phi-\phi(y)] \dot{x}$ along $\Gamma_{y}$, with some $\lambda \in \mathbb{R}$. As $p_{y} \in K \subseteq \phi^{-1}(0)$, we have $\phi(x(1))=\phi\left(p_{y}\right)=0$, and so $\dot{x}(1)$ is an eigenvector of $\nabla v_{x(1)}$ for the eigenvalue $\lambda_{y}=\lambda-\phi(y)$. Since $y \rightarrow z$, a subsequence of the tangent directions of $\Gamma_{y}$ at $p_{y}$ tends to a limit, which must lie in Ker $\nabla v_{z} \subseteq T_{z} M$ (see Remark 5.1(ii)), so that, for the subsequence, $\lambda_{y} \rightarrow 0$ as $y \rightarrow z$. At the same time, according to Lemma 12.2(b-iii) applied to the geodesic segment $\Gamma \subseteq K$ joining $z$ to $p_{y}$, each $\lambda_{y}$ is an eigenvalue of $\nabla v_{z}$, and finiteness of the spectrum of $\nabla v_{z}$ gives $\lambda_{y}=0$ for all but finitely many $y$ in the subsequence. For such $y$, the equality $\nabla_{\dot{x}} v=[\lambda+\phi-\phi(y)] \dot{x}$ reads $\nabla_{\dot{x}} v=\phi \dot{x}$. Lemma 12.4(i) now implies that $\phi=0$ at some interior point of each $\Gamma_{y}$ in question, contrary to the last paragraph.

Lemma 14.2. Let $M, g, v, Z, z, \phi, H$ be as in Theorem C. If $H \subseteq T_{z} M$ is a null subspace, $\phi(z)=0$, and $\nabla \phi_{z} \notin \nabla v_{z}\left(T_{z} M\right)$, then $Z \cap U^{\prime}=\exp _{z}[H \cap U]$ for any sufficiently small star-shaped neighborhood $U$ of 0 in $T_{z} M$ mapped by $\exp _{z}$ diffeomorphically onto a neighborhood $U^{\prime}$ of $z$ in $M$.

Proof. As $H$ is a null subspace, $H \subseteq H^{\perp}$. Thus, $H \backslash V=\varnothing$ for $V=H \cap H^{\perp}$, and so, by in Lemma 14.1, $\phi=0$ at all zeros of $v$ near $z$. On the other hand, for $U, U^{\prime}$ as 
above, Lemma 13.1(a) states that $K=\exp _{z}[H \cap U]$ is a submanifold of $U^{\prime}$ contained in both $\phi^{-1}(0)$ and the zero set $Z$ of $v$. For sufficiently small $U, U^{\prime}$ and a submanifold $N$ of $M$ chosen as in Lemma 11.1, we now have $K \subseteq Z \cap U^{\prime} \subseteq N \cap \phi^{-1}(0)$. Since $\phi(z)=0$ and $\nabla \phi_{z} \notin \nabla v_{z}\left(T_{z} M\right)$, Lemma 9.1(b) implies that $\nabla \phi_{z}$ is not orthogonal to the whole space $\mathcal{B}_{z}=\operatorname{Ker} \nabla v_{z}$. Thus, $d \phi_{z}$ is not identically zero on $\mathcal{B}_{z}$, and $H=\mathcal{B}_{z} \cap \operatorname{Ker} d \phi_{z}$ is a codimension-one subspace of $\mathcal{B}_{z}$. As $H=T_{z} K$ and $T_{z} N=\mathcal{B}_{z}$ (see Lemma 11.1(ii)), $K$ is a codimension-one submanifold of $N$, and the restriction of $\phi$ to $N$ has a nonzero differential at $z$. Consequently, applying Lemma 2.1(a) to $\beta=\phi$, we can make $N, U$ and $U^{\prime}$ even smaller, so as to have $K=N \cap \phi^{-1}(0)$, which proves our assertion since $K \subseteq Z \cap U^{\prime} \subseteq N \cap \phi^{-1}(0)$.

\section{Proof of Theorem C, case (2,a)}

Let $U$ be a star-shaped neighborhood of 0 in $T_{z} M$ mapped by $\exp _{z}$ diffeomorphically onto a neighborhood $U^{\prime}$ of $z$ in $M$, such that $\phi \neq 0$ everywhere in $U^{\prime}$. For every $x \in\left(Z \cap U^{\prime}\right) \backslash\{z\}$, we denote by $L_{x}$ the tangent direction at $z$ of the geodesic segment $\Gamma_{x}$ joining $z$ to $x$ in $U^{\prime}$. Then

$$
\Gamma_{x} \text { is null and } L_{x} \subseteq \mathcal{B}_{z}=\operatorname{Ker} \nabla v_{z} \text { for all } x \in\left(Z \cap U^{\prime}\right) \backslash\{z\},
$$

provided that $U$ and $U^{\prime}$ are chosen small enough. In fact, $\Gamma_{x}$ is null by Remark 12.1, Lemma 12.3(b) in turn shows that $v$ is tangent to $\Gamma_{x}$, and hence so is the covariant derivative of $v$ in the direction of $\Gamma_{x}$. Thus, each $L_{x}$ is contained in the eigenspace of $\nabla v_{z}$ for some eigenvalue $\lambda_{x}$. If, no matter how small one made $U$ and $U^{\prime}$, the inclusion in (29) failed to hold, there would exist a sequence, converging to $z$, of points $x \in\left(Z \cap U^{\prime}\right) \backslash\{z\}$ with $\lambda_{x} \neq 0$. Passing to a subsequence, we would have $L_{x} \rightarrow L$ for some line $L$ through 0 in $T_{z} M$. As $L$ would then be a radial limit direction of $Z$ at $z$ (cf. the end of Section 5), Remark 5.1(ii) with $\psi=v$ would imply that $L \subseteq \operatorname{Ker} \nabla v_{z}$, and so $\lambda_{x} \rightarrow 0$. Finiteness of the spectrum of $\nabla v_{z}$ would now give $\lambda_{x}=0$ for all but finitely many $x$ in the subsequence, contrary to how the subsequence was selected.

On the other hand, by Lemma 9.1(a),

$$
\text { both } \mathcal{B}_{z}=\operatorname{Ker} \nabla v_{z} \text { and } H \subseteq \mathcal{B}_{z} \text { are null subspaces of } T_{z} M \text {. }
$$

If $\mathcal{B}_{z}$ is contained in $\operatorname{Ker} d \phi_{z}$, so that $H=\mathcal{B}_{z}$, (29) yields (28), with $C \cap H=H$ in view of (30), which, according to Remark 13.2, proves Theorem $\square$ when $\mathcal{B}_{z} \subseteq \operatorname{Ker} d \phi_{z}$.

From now on we therefore assume that $\mathcal{B}_{z}$ is not contained in Ker $d \phi_{z}$. Thus, $H$ is a codimension-one subspace of $\mathcal{B}_{z}$, and $K=\exp _{z}[H \cap U]$ is a codimension-one submanifold of $N=\exp _{z}\left[\mathcal{B}_{z} \cap U\right]$, while the restriction of $\phi$ to $N$ has a nonzero differential at $z$. In addition, by Lemma 13.1(a) and (30), $\phi=\phi(z)$ everywhere in $K$. According to Lemma 2.1(a) for $\beta=\phi-\phi(z)$, making $U$ and $U^{\prime}$ even smaller if necessary, we can ensure that $\phi \neq \phi(z)$ everywhere in $N \backslash K$. This shows that no zero $x$ of $v$ lies in $N \backslash K$, for if one did, Lemma 12.4(ii) and (29) would give $\phi=\phi(z)$ somewhere in $\Gamma_{x} \backslash\{z\} \subseteq N \backslash K$. In other words, we again have (28), with $C \cap H=H$ (cf. (30)), which, in view of Remark 13.2, proves Theorem C in case (2.a). 


\section{Proof of Theorem $\mathrm{C}$, case (2,b)}

We are free to assume that $\mathcal{B}_{z}=\operatorname{Ker} \nabla v_{z}$ is not a null subspace of $T_{z} M$. Namely, if $\mathcal{B}_{z} \subseteq T_{z} M$ is a null subspace, then so is $H \subseteq \mathcal{B}_{z}$, and the assertion of Theorem $\mathrm{C}$ is immediate from Lemma 14.2, with $C \cap H=H$ since $H$ is null.

Let us choose the submanifolds $N$ and $K$ of $M$ as in Lemmas 11.1 and 13.1, with $U$ small enough so as to ensure that $K \subseteq N$. Such $U$ must exist since, by Lemma 13.1(a), $K$ is contained in zero set $Z$ of $v$, while all zeros of $v$ close to $z$ lie in $N$. Lemmas 13.1(a),(d) and 11.1(ii) imply that, in fact, not only $v_{x}=0$, but also $T_{x} N=\operatorname{Ker} \nabla v_{x}$ whenever $x \in K$. We may now use a local trivialization of $T M$ on $\exp _{z}(U)$ to identify each tangent space $T_{x} M$, for $x \in N$, with $T_{z} M$, in such a way that $T_{z} M$ itself remains unchanged. (One could for instance use the identifications provided by parallel transports along geodesics emanating from z.) This allows us to treat $2 v$ as a vector-valued function $f: N \rightarrow T_{z} M$.

The hypotheses of Theorem 7.5 are now satisfied by our $K, N, z$, the vector space $\mathcal{T}=T_{z} M$ with $\langle\rangle=,g_{z}$ and $f$ as above, $\phi: N \rightarrow \mathbb{R}$ obtained by restricting to $N$ the function in (1), and $Y=\exp _{z}[C \cap H \cap U]$, where $H=\operatorname{Ker} \nabla v_{z} \cap \operatorname{Ker} d \phi_{z} \subseteq T_{z} M$ and $C=\left\{u \in T_{z} M: g_{z}(u, u)=0\right\}$ is the null cone, provided that one replaces $N, K$ and $U$ with suitable smaller neighborhoods of $z$ in $N$ or $K$ and 0 in $T_{z} M$.

Specifically, $d \phi_{z}$ is not identically zero on $T_{z} N=\operatorname{Ker} \nabla v_{z}$ since $\phi(z)=0$ and $\nabla \phi_{z} \notin \nabla v_{z}\left(T_{z} M\right)$ by (2.b), and so, in view of Lemma 9.1(b), $\nabla \phi_{z}$ is not orthogonal to all of $\operatorname{Ker} \nabla v_{z}$. Next, $Y$ is a quadric of the required kind due to the very definition of a quadric, in the lines preceding Lemma [7.2, with the rôle of $\Psi$ played here by the restriction of $\exp _{z}$ to $H \cap U$, which sends $H \cap U$ into $P=N \cap \phi^{-1}(0)$ according to Lemma 13.1(a), and is a diffeomorphism for dimensional reasons. Condition (a) in Theorem 7.5 holds in turn due to the assumption about $\mathcal{B}_{z}=T_{z} N$ made at the beginning of this section, condition (b) follows since $K=\exp _{z}[V \cap U]$ for $V=H \cap H^{\perp}$ (see Lemma 13.1), and (c) is immediate from Lemma 13.1)(a) (which states that $v=0$, and hence $f=0$, on $Y$ ), combined with the equality $T_{x} N=\operatorname{Ker} \nabla v_{x}$ for $x \in K$, established above (which amounts to $d f=0$ everywhere in $K$ ). Lemma 11.1(iv) now implies that the left-hand side in (d) equals the Hessian at $z$ of the function $y \mapsto\langle w, f(y)\rangle$ on $N$.

In view of (2. b), the assertion of Theorem 7.5)(ii) amounts to the assumption of Lemma 14.1, which now implies that all zeros of $v$ close to $z$ lie in $P=N \cap \phi^{-1}(0)$. By Theorem $7.5(\mathrm{i})$, they must lie in $Y$ as well, and so (28) holds for sufficiently small $U$ and $U^{\prime}$. Combined with Remark 13.2, this proves Theorem $\mathrm{C}$ in case (2. b).

\section{Proof of Theorem A}

Let us fix a point $z \in Z$. We denote by $\phi$ the function in (1), by $H$ the subspace $\operatorname{Ker} \nabla v_{z} \cap \operatorname{Ker} d \phi_{z}$ of $T_{z} M$, and by $T_{z} Z$ (or, $b_{z}$ ) the tangent space (or, respectively, the second fundamental form) at $z$ of the connected component of $Z$ containing $z$, provided that $z$ is not a singular point of $Z$. Three cases are possible: 
(i) neither (2, a) nor (2,b) holds at $z$,

(ii) $z$ satisfies (2,a) or (2,b) and the metric $g_{z}$ restricted to $H$ is not semidefinite,

(iii) $z$ satisfies (2,a) or (2,b) and $g_{z}$ is semidefinite on $H$.

In case (i), Theorem $\mathrm{B}$ allows us to change the metric conformally so as to make $v$ a Killing field for the new metric $g^{\prime}$ on some normal-coordinate neighborhood $U^{\prime}$ of $z$ in $\left(M, g^{\prime}\right)$. Assertion (a) in Theorem $\mathrm{A}$ is now immediate, as $\exp _{z}$ (corresponding to $g^{\prime}$ ) sends short line segments emanating from 0 in $T_{z} M$ onto $g^{\prime}$-geodesics, and so the local flow of $v$ corresponds via $\exp _{z}$ to the linear local flow near 0 in $T_{z} M$, generated by $\partial v_{z}$ (notation of Section 3 ).

Consequently, in case (i), $T_{z} Z=\operatorname{Ker} \partial v_{z}=\operatorname{Ker} \nabla v_{z}$. Also, since the $g^{\prime}$-Killing field $v$ has zero $g^{\prime}$-divergence, $\nabla v_{x}=\partial v_{x}$ is traceless at every $x \in Z$ near $z$. Thus, $\phi=0$ at all points of $Z$ close to $z$. As a result, the codimension of $T_{z} Z$ in $T_{z} M$ is even (Lemma 9.1(b)), while $b_{z}$ is a tensor multiple of the metric due to Lemma 2.2(ii), the already-established assertion (a) of Theorem $\mathrm{A}$, and (5).

Next, in cases (ii) and (iii), Theorem Clearly implies (b) in Theorem A, with $g^{\prime}=g$, while Lemma 13.1(a) shows that $\phi=\phi(z)$ at all points of $Z$ close to $z$. Combined with Remarks 6.1 and 6.2, this gives the description of the singular subset $\Delta$ required in Theorem A. Thus, in case (ii) (or, (iii)), $z$ is a singular (or, respectively, nonsingular) point of $Z$.

Consider now case (iii). In view of Theorem $\mathrm{C}, T_{z} Z$ is the nullspace of $H$, that is, $T_{z} Z=H \cap H^{\perp}$, while $b_{z}=0$ by Lemma 2.2(ii) and, as noted above, $Z$ has no singularities near $z$. It follows now that case (iii) represents an open condition, or, in other words, we will still have (iii) after $z$ has been replaced with any nearby point $x \in Z$. In fact, case (ii) for such $x$ cannot occur since they are nonsingular in $Z$. To exclude case (i) for them, note that (iii), for $z$, has two subcases: (2,a) and (2), b). In the former, (2,a) obviously remains valid at nearby points. The latter subcase amounts in turn to assuming that $\phi(z)=0$ and $\operatorname{Ker} \nabla v_{z}$ is not contained in Ker $d \phi_{z}$ (see Lemma 9.1(b)). By Lemma 13.1(a),(c),(d), these assumptions will still hold when $z$ is replaced with any nearby $x \in K$, which, by Theorem $\mathbb{C}$, are the same points as nearby $x \in Z$. Consequently, points $x \in Z$ near $z$ cannot represent case (i).

Thus, in case (iii), due to its open-condition property, the equalities $T_{z} Z=H \cap H^{\perp}$ and $b_{z}=0$ imply that the intersection of $Z$ with some neighborhood of $z$ is a null totally geodesic submanifold of $(M, g)$. The proof of Theorem $\mathrm{A}$ is now complete.

REMARK 17.1. As we just saw, case (iii) constitutes an open condition in the set $Z$ of all zeros of $v$. By Theorem $\mathrm{B}$, the same is true of case (i). Not so, however, in case (ii): according to Theorem $\mathrm{C}$ and Remark 6.2(b), in every neighborhood of a point $z \in Z$ representing case (ii), there exist points of $Z$ which are nonsingular, and hence, as we saw above, must correspond to case (i) or case (iii).

REMARK 17.2. In the Lorentzian case, Theorem A can obviously be rephrased so as to reflect the fact that null submanifolds can be at most one-dimensional, while the 
only singularities of the zero set that may occur are those associated with null cones in Lorentzian subspaces of the tangent space.

REMARK 17.3. Theorem A provides hardly any information about those connected components of $\left(Z \cap U^{\prime}\right) \backslash \Delta$ which happen to be one-dimensional. For submanifolds $K$ with $\operatorname{dim} K=1$, the property of being totally umbilical is nearly meaningless, as such $K$ always has it, except at points $x \in K$ at which $T_{z} K$ is a null subspace and the second fundamental form $b_{x}$ is nonzero. It is worth pointing out that one-dimensional connected components of $\left(Z \cap U^{\prime}\right) \backslash \Delta$ need not, in general, be conformal circles. (For a definition, see [1].) In fact, a non-null geodesic $t \mapsto x(t)$ in a pseudo-Riemannian manifold is a conformal circle if and only if $S(\dot{x}, \cdot)=0$, where $S$ is the Schouten tensor [1, p. 217]. Let $u$ now be a Killing field with a nonempty discrete set $Y$ of zeros on a pseudoRiemannian manifold $(N, h)$, the scalar curvature of which is nonzero at some point $y \in Y$. (Such $u$ obviously exist on even-dimensional standard spheres.) Extending $u$ trivially to a Killing field $v$ on the product manifold $(M, g)=\left(\mathbb{R}, d t^{2}\right) \times(N, h)$, we see that the geodesic $\mathbb{R} \ni t \mapsto(t, y)$ forms a connected component of the zero set of $v$, while $\operatorname{Ric}(\dot{x}, \cdot)=0$, and hence $S(\dot{x}, \cdot) \neq 0$ due to the definition of $S$ in Section 9 . Thus, the geodesic in question is not a conformal circle.

\section{References}

[1] Bailey T N and Eastwood M G 1990 Proc. Amer. Math. Soc. 108215

[2] Banyaga A and Hurtubise D E (2004) Expo. Math. 22365.

[3] Beig R 1992 Conformal Killing vectors near a fixed point, Institut für Theoretische Physik, Universität Wien, unpublished manuscript

[4] Belgun F, Moroianu A and Ornea L 2010 Preprint arXiv:1002:0482v3

[5] Blair D E 1974 Nagoya Math. J. 551

[6] Capocci M S 1999 Class. Quantum Grav. 16927

[7] Cartan E 1923 C. R. Math. Acad. Sci. Paris 174857

[8] Cartan E 1923 Ann. Soc. Polon. Math. 2171

[9] Derdzinski A and Roter W 2007 Tohoku Math. J. (2) 59565

[10] Frances C 2008 Essential conformal structures in Riemannian and Lorentzian geometry Recent Developments in Pseudo-Riemannian Geometry eds D V Alekseevsky and H Baum (ESI Lect. Math. Phys. EMS Zürich) pp 231-260

[11] Kobayashi S 1958 Nagoya Math. J. 1363

[12] Ogiue K 1967 Kōdai Math. Sem. Rep. 19193 